\title{
A High-Accuracy Eulerian Gyrokinetic Solver for Collisional Plasmas
}

\author{
J. Candy ${ }^{1}$, E.A. Belli ${ }^{1}$ and R.V. Bravenec ${ }^{2}$ \\ ${ }^{1}$ General Atomics, P.O. Box 85608, San Diego, CA 92186-5608, USA \\ ${ }^{3}$ Fourth State Research, Austin, TX, USA
}

\begin{abstract}
We describe a new approach to solve the electromagnetic gyrokinetic equations which is optimized for accurate treatment of multispecies Fokker-Planck collisions including both pitch-angle and energy diffusion. The new algorithm is spectral/pseudospectral in four of the five phase space dimensions, and in the fieldline direction a novel 5th-order conservative upwind scheme is used to permit high-accuracy electromagnetic simulation even in the limit of very high plasma $\beta$ and vanishingly small perpendicular wavenumber, $k_{\perp} \rightarrow 0$. To our knowledge, this is the first pseudospectral implementation of the collision operator in a gyrokinetic code. We show that the new solver agrees closely with GYRO in the limit of weak Lorentz collisions, but gives a significantly more realistic description of collisions at high collision frequency. The numerical methods are also designed to be efficient and scalable for multiscale simulations that treat ion-scale and electron-scale turbulence simultaneously.
\end{abstract}

Keywords: gyrokinetic, Eulerian, Continuum

PACS: 52.25.Dg, 52.25.Fi, 52.65.-y

\section{Introduction}

For gyrokinetic studies of plasma microturbulence in the H-mode pedestal and L-mode edge, collisions are expected to play a more critical role than in the core due to the lower electron and ion temperatures, and correspondingly higher collision frequencies. Here the $\delta f$ approximation is formally valid for electron-scale fluctuations as well as for ion-scale fluctuations where the ion gyroradius is small compared to the equilibrium length scale, as long as the simulation region is far enough away from the separatrix so that orbit loss is not significant. The dominant collisional process in gyrokinetics is the detrapping of electrons, which reduces trapped-electron drive and thereby lowers linear instability growth rates. A very weak, secondary collisional process is the ion-ion collisional damping of zonal flows. We believe it is fair to suggest that existing Eulerian gyrokinetic codes use a numerical mesh that is motivated by, and optimized for, the collisionless equations rather than for accurate treatment of the collision operator. Thus, for pedestal-relevant studies, a significant improvement in gyrokinetic solvers may be realized by including more advanced gyrokinetic collision operators together with numerical algorithms optimized for their discretization in the strong-collisionality regime.

In neoclassical transport studies, comparative studies have shown that model operators can yield inaccuracies - compared with the full linearized Fokker-Planck operator - by as large as 10$15 \%$ for the neoclassical particle fluxes and $20-30 \%$ for the neoclassical ion energy fluxes [1]. On the other hand, gyrokinetic studies of the accuracy and limitations of commonly used model operators

Email address: candy@fusion.gat.com (J. Candy ${ }^{1}$, E.A. Belli ${ }^{1}$ and R.V. Bravenec ${ }^{2}$ ) 

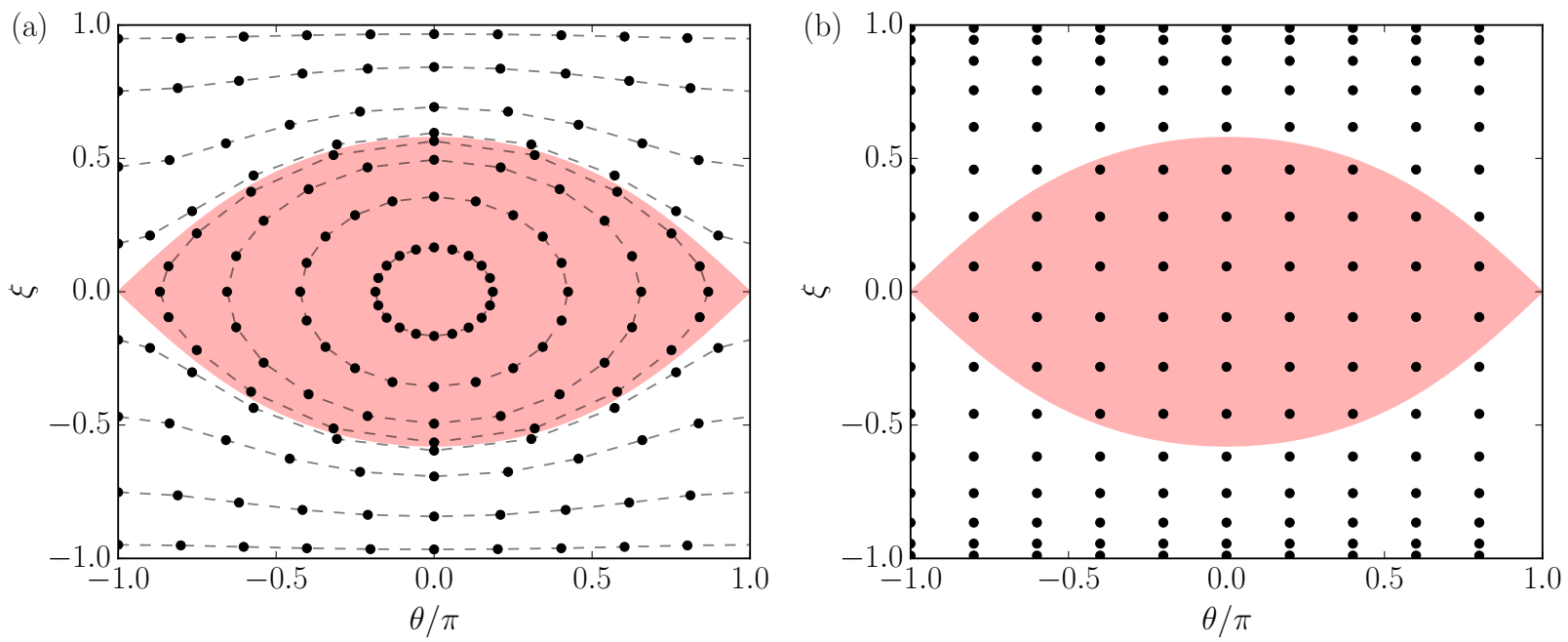

Figure 1: GYRO irregular $(\theta, \xi)$ mesh (a) showing constant-of-motion alignment, versus CGYRO regular $(\theta, \xi)$ mesh (b). In the GYRO mesh, dashed curves denote contours of constant $\mu$, such that a given collisionless unperturbed particle orbit is confined to single curve. The GYRO mesh is ideal for the collisionless case, whereas the CGYRO mesh becomes progressively more suitable for discrectization of collisional plasmas. The shaded area corresponds to the trapped-particle region $\xi^{2}<1-B(\theta) / B(\pi)$.

have been much more limited in scope. Early nonlinear gyrokinetic simulations included collisions with a relatively simple Lorentz pitch-angle scattering operator. Advanced operators including energy diffusion have recently been implemented in GS2 [2], GENE [3], and GKW [4]. However, the accuracy of the model operators and the convergence properties of their numerical implementations have not been well-explored. With GS2, it was shown in simplified Z-pinch geometry that energy diffusion suppresses the short wavelength structure beyond that due to pitch angle scattering alone [2]. Some detailed studies in tokamak geometry were done with GKW, showing that energy diffusion enhances the stabilization of trapped electron modes (TEM) [4].

Motivated by these considerations, a new gyrokinetic solver CGYRO has been developed for more accurate treatment of advanced collision operators in high collisionality regimes. Building on experience with the neoclassical code NEO [5, 1], CGYRO uses the $(\xi, v)$ coordinate system, where $\xi=v_{\|} / v$ is the cosine of the pitch angle, and $v$ is the speed. These coordinates simplify the analytic form of the collision operator and thereby permit the implementation of high-order numerical schemes to treat multi-species collisions and energy diffusion. As early as 1957 [6], these coordinates were pointed out as being particularly useful for respresentation of the Fokker-Planck collision operator.

As already noted, existing gyrokinetic codes use velocity-space coordinates optimized for the collisionless problem. For example, GYRO [7] and GS2 [8,9] use $(\lambda, \varepsilon)$, where $\lambda=v_{\perp}^{2} /\left(v^{2} B\right)$ and $\varepsilon=m_{a} v^{2} /\left(2 T_{a}\right)$. These coordinates are in fact unperturbed, collisionless constants of motion. The main advantage of the constant-of-motion coordinates is that the poloidal $(\theta)$ discretization is aligned with particle orbits, so that there is no need to take $\theta$-derivatives across the trapped-passing (TP) boundary. This feature of the $(\lambda, \varepsilon)$ coordinate system is illustrated in Fig. 1a, and compared with the $(\xi, v)$ system in Fig. 1b. For early - typically collisionless - gyrokinetic simulation, this was viewed as a critical feature of the algorithm since the collisionless electron distribution function is not continuous across the TP boundary. Conversely, the significant disadvantage of these coordinates is that it is very difficult to accurately discretize the collision operator since the constant-of-motion 
coordinates map to an irregular grid in $(\xi, \theta)$. To this end, GYRO uses a radial basis function interpolant in the $(\xi, \theta)$ plane [10]. Relatedly, the codes GENE [11] and GKW [12] use $\left(\mu, v_{\|}\right)$ coordinates, where $\mu=v_{\perp}^{2} /(2 B)$. The latter coordinates are the standard ones used for the Lietransform derivation of the collisionless gyrokinetic equations [13]. Once again, although convenient for the collisionless case, $\left(\mu, v_{\|}\right)$coordinates are not ideal for evaluating the collision operator because they generate cross-derivatives, and lead to issues with constructing suitable boundary conditions. If instead we restrict the domain of interest to collisional plasmas for which the electron distribution is expected to be sufficiently smooth across the TP boundary, then the $(\xi, v)$ coordinates make it possible to implement elegant and spectrally-accurate discretization schemes. In this way, CGYRO builds upon the success of NEO by implementing spectrally accurate collision operators in $(\xi, v)$ coordinates to describe inter-species collisions between particles with arbitrary masses.

The rest of this paper is organized as follows. In Section 2, the basic simulation equations, including the collision models, are described. In Section 3, details of the numerical discretization algorithms are given. Numerical results are presented in section 4, including benchmarks with GYRO and a comparison of reduced collision models on gyrokinetic nonlinear transport.

\section{Theoretical formulation and simulation equations}

\subsection{Equilibrium geometry}

We adopt the non-orthogonal field-aligned coordinate system $(\psi, \theta, \alpha)$, together with the Clebsch representation for the magnetic field [14]:

$$
\mathbf{B}=\nabla \alpha \times \nabla \psi \quad \text { such that } \quad \mathbf{B} \cdot \nabla \alpha=\mathbf{B} \cdot \nabla \psi=0 .
$$

The angle $\alpha$ is written in terms of the toroidal angle $\varphi$ as $\alpha \doteq \varphi+\nu(\psi, \theta)$. In Eq. (1), $\psi$ is the poloidal flux divided by $2 \pi$, and $\theta$ simultaneously refers to an angle in the poloidal plane (at fixed $\varphi$ ) and a parameterization of distance along a field line (at fixed $\alpha$ ). In these coordinates, the Jacobian determinant $\mathcal{J}_{\psi}$ satisfies

$$
\mathcal{J}_{\psi} \doteq \frac{1}{\nabla \psi \times \nabla \theta \cdot \nabla \alpha}=\frac{1}{\nabla \psi \times \nabla \theta \cdot \nabla \varphi}
$$

We write the magnetic field as

$$
\mathbf{B}=\nabla \varphi \times \nabla \psi+I(\psi) \nabla \varphi .
$$

The function $\nu(\psi, \theta)$ can be related to the current function $I$ according to

$$
\nu(\psi, \theta)=-I(\psi) \int_{0}^{\theta} \mathcal{J}_{\psi}|\nabla \varphi|^{2} d \theta
$$

In Eq. (3), $B_{t}=I(\psi) / R$ is the toroidal field strength and $B_{p}=|\nabla \psi| / R$ is the poloidal field strength. We define $\mathbf{b}$ as the unit vector in the direction of $\mathbf{B}$. The flux-surface average of a function $f$ is given by

$$
\langle f\rangle \doteq \frac{\oint d \theta d \varphi \mathcal{J}_{\psi} f}{\oint d \theta d \varphi \mathcal{J}_{\psi}}
$$




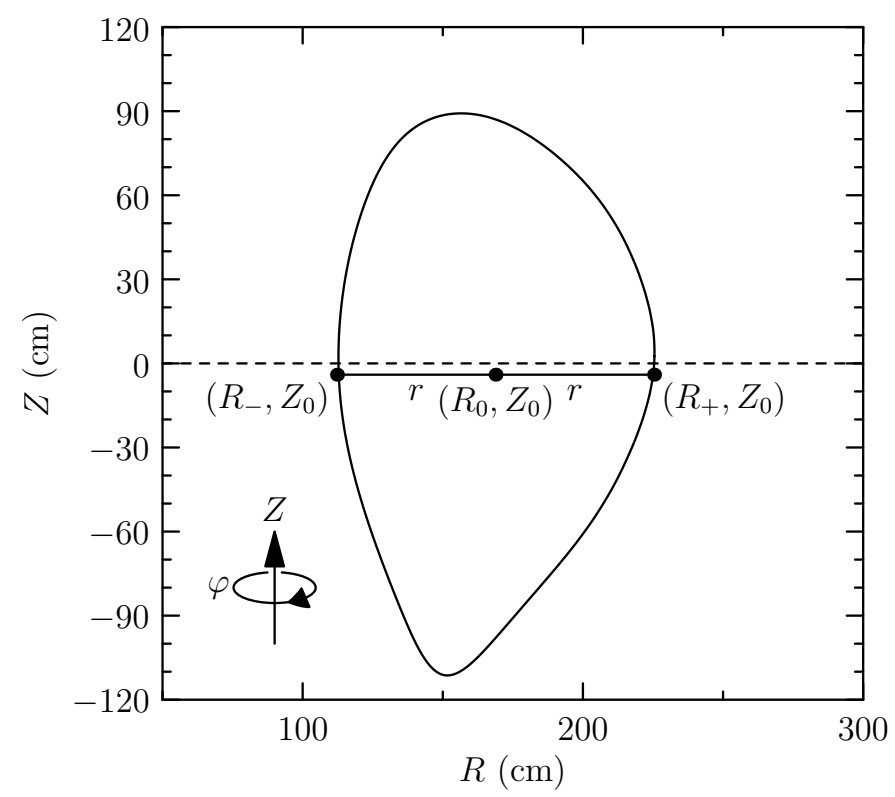

Figure 2: Illustration of minor and major radii, $r$ and $R_{0}$, of an arbitrary flux surface, where $Z=Z_{0}$ is the elevation of the centroid.

\subsection{Units, Conventions, and Definitions}

Unless otherwise specified, the conventions and units defined in this section are used throughout the paper. The midplane minor radius $r$ which measures the half-width of the flux surface at the elevation of the centroid [15]. Similarly, we define $R_{0}$ as the major radius of the flux surface at the elevation of the centroid. These can be defined precisely as

$$
r \doteq \frac{R_{+}-R_{-}}{2} \text { and } R_{0} \doteq \frac{R_{+}+R_{-}}{2}
$$

where $R_{+}$and $R_{-}$are the points of intersection of the flux surface with the line $Z=Z_{0}$. Here, $Z_{0}$ is the elevation of the centroid. These quantities are illustrated in Fig. 2. Length is measured in units of $a$, the midplane minor radius of the last closed flux surface. The inverse aspect ratio is $\epsilon=r / R_{0}$. Frequencies are measured in units of $c_{s} / a$, where $c_{s}=\sqrt{T_{e} / m_{\mathrm{D}}}$ is the deuteron sound speed, $T_{e}$ is the electron temperature, and $m_{\mathrm{D}}$ is the deuteron mass. It is also useful to define the species-dependent thermal speed, gyrofrequency and gyroradius, respectively, as

$$
v_{t a} \doteq \sqrt{T_{a} / m_{a}}, \quad \Omega_{c a} \doteq \frac{z_{a} e B}{m_{a} c}, \quad \text { and } \quad \rho_{a} \doteq \frac{v_{t a}}{\Omega_{c a}}
$$

where the subscript $a$ denotes the species index. Next, we introduce an effective magnetic field $B_{\text {unit }}[15,16]$, which is defined with reference to a global equilibrium through the relation

$$
B_{\text {unit }}(r)=\frac{q}{r} \psi^{\prime}
$$

where $\psi^{\prime}$ is defined as $\partial \psi / \partial r$, and $q$ is the safety factor

$$
q(\psi) \doteq \frac{1}{2 \pi} \int_{0}^{2 \pi} \frac{\mathbf{B} \cdot \nabla \varphi}{\mathbf{B} \cdot \nabla \theta} d \theta=\frac{1}{2 \pi} \int_{0}^{2 \pi}\left(-\frac{\partial \nu}{\partial \theta}\right) d \theta=\frac{\nu(\psi, 0)-\nu(\psi, 2 \pi)}{2 \pi} .
$$


This flux function $B_{\text {unit }}$ provides a convenient normalizing (or unit of) magnetic field. In terms of $B_{\text {unit }}$, we define an effective electron beta and an effective ion-sound gyroradius as

$$
\beta_{e, \text { unit }} \doteq \frac{8 \pi n_{e} T_{e}}{B_{\text {unit }}^{2}} \quad \text { and } \quad \rho_{s, \text { unit }} \doteq \frac{c_{s}}{e B_{\text {unit }} /\left(m_{D} c\right)} .
$$

Hereafter, we will write the effective ion-sound gyroradius as $\rho_{s}$ instead of $\rho_{s, \text { unit }}$ for brevity. This notation is consistent with that in Ref. [17]. Finally, we can define the effective pressure gradient as

$$
\beta^{*} \doteq-\frac{8 \pi}{B_{\text {unit }}^{2}} \frac{d p}{d r}
$$

where $p=\sum_{a} n_{a} T_{a}$ is the total plasma pressure. The temperature and density gradient inverse scale lengths are

$$
\frac{1}{L_{T a}}=-\frac{d \ln T_{a}}{d r} \text { and } \quad \frac{1}{L_{n a}}=-\frac{d \ln n_{a}}{d r} .
$$

For nonlinear simulations, because the energy fluxes have a natural gyroBohm scaling, we normalize them to a reference gyroBohm level

$$
Q_{G B}=n_{e} T_{e} c_{s} \frac{\rho_{s}^{2}}{a^{2}}
$$

Finally, the inter-particle collision rate is defined as

$$
\tau_{a b}^{-1}=\frac{\sqrt{2} \pi e^{4} z_{a}^{2} z_{b}^{2} n_{b}}{m_{a}^{1 / 2} T_{a}^{3 / 2}} \ln \Lambda .
$$

Rather than the general $a-b$ collision rate above, it is useful to define an simpler collision rate to parameterize simulations. For this purpose we introduce the dimensionless electron collision frequency

$$
\bar{\nu}_{e} \doteq \frac{a}{c_{s}} \tau_{e e}^{-1}=\frac{a}{c_{s}} \frac{\sqrt{2} \pi e^{4} n_{e}}{m_{e}^{1 / 2} T_{e}^{3 / 2}} \ln \Lambda .
$$

The collision dependence of all scans reported in the present work are plotted as a function of this parameter.

\subsection{The gyrokinetic equation}

We consider the formulation of the electromagnetic gyrokinetic equation in $\left(r, \theta, \alpha, \xi, u_{a}\right)$ coordinates. Here the velocity-space coordinates are the cosine of the pitch angle, $\xi \doteq v_{\|} / v$, and the

normalized velocity, $u_{a}=v /\left(\sqrt{2} v_{t a}\right)$. The spatial coordinates refer to the position of the guiding center $\mathbf{R}=(r, \theta, \alpha)$. The nonlinear gyrokinetic equation [18, 19] for species $a$ takes the form

$$
\frac{\partial h_{a}}{\partial t}-i\left(\omega_{\theta}+\omega_{\xi}+\omega_{\mathrm{d}}\right) H_{a}+\frac{c}{\psi^{\prime}}\left[f_{0 a}+h_{a}, \Psi_{a}\right]=\sum_{b} C_{a b}^{\mathrm{GK}}
$$

where $f_{0 a}$ is the zeroth-order equilibrium distribution function, which has the Maxwellian form

$$
f_{0 a}=\frac{n_{a}}{\left(2 \pi v_{t a}^{2}\right)^{3 / 2}} e^{-u_{a}^{2}}
$$

In this work, for brevity, we consider plasma rotation to be at the diamagnetic level. This justifies the use of a stationary Maxwellian equilibrium. In future work we will implement sonic rotation [19] 
in a manner analogous to that in NEO [20]. The inclusion of sonic rotation in CGYRO, in particular centrifugal terms, is facilitated by the use of $(\xi, v)$ coordinates and was a further motivation for their use.

The derivation of Eq. (16) assumes that all fluctuating quantities have the eikonal form

$$
z(\mathbf{R})=\sum_{\mathbf{k}_{\perp}} e^{i S(\mathbf{R})} z_{\mathbf{k}_{\perp}}
$$

where the perpendicular wavenumber is $\mathbf{k}_{\perp} \doteq \nabla_{\perp} S$. The function $H_{a}$ is the non-adiabatic distribution,

$$
H_{a}\left(\mathbf{R}, \xi, u_{a}\right) \doteq h_{a}\left(\mathbf{R}, \xi, u_{a}\right)+\frac{z_{a} e f_{0 a}}{T_{a}} \Psi_{a}\left(\mathbf{R}, \xi, u_{a}\right),
$$

whereas $h_{a}$ is more a convenient variable for time evolution. $\Psi_{a}$ is the field potential:

$$
\Psi_{a}\left(\mathbf{R}, \xi, u_{a}\right) \doteq G_{0 a}\left[\delta \phi(\mathbf{R})-\frac{v_{\|}}{c} \delta A_{\|}(\mathbf{R})\right]+\frac{v_{\perp}^{2}}{\Omega_{c a} c} G_{1 a} \delta B_{\|}(\mathbf{R}) .
$$

Above, $G_{0 a}$ and $G_{1 a}$ are linear operators arising from gyro-averaging [19], with the familiar spectral representations in terms of Bessel functions:

$$
\begin{aligned}
G_{0 a} z(\mathbf{R}) & =\sum_{\mathbf{k}_{\perp}} e^{i S(\mathbf{R})} J_{0}\left(\gamma_{a}\right) z_{\mathbf{k}_{\perp}} \\
G_{1 a} z(\mathbf{R}) & =\sum_{\mathbf{k}_{\perp}} e^{i S(\mathbf{R})} \frac{J_{1}\left(\gamma_{a}\right)}{\gamma_{a}} z_{\mathbf{k}_{\perp}}
\end{aligned}
$$

such that $\gamma_{a} \doteq k_{\perp} v_{\perp} / \Omega_{c a}$. In this notation, the fluctuating part of the perturbed particle distribution takes the form

$$
f_{1 a}\left(\mathbf{R}, \xi, u_{a}\right)=\sum_{\mathbf{k}_{\perp}} e^{i S(\mathbf{R})} f_{1 a, \mathbf{k}_{\perp}}\left(\xi, u_{a}\right)
$$

where each spectral component is the sum of a gyroangle-independent term and a gyroharmonic term:

$$
f_{1 a, \mathbf{k}_{\perp}} \doteq-\frac{z_{a} e f_{0 a}}{T_{a}} \delta \phi_{\mathbf{k}_{\perp}}+e^{-i \mathbf{k}_{\perp} \cdot \boldsymbol{\rho}_{a}} H_{a, \mathbf{k}_{\perp}}
$$

Here, $\boldsymbol{\rho}_{a}=\boldsymbol{\rho}_{a}(\vartheta)$ is the gyroradius vector and $\vartheta$ is the gyroangle. In Eq. (16), we have defined the parallel streaming operator

$$
-i \omega_{\theta}=v \frac{\xi}{\mathcal{J}_{\psi} B} \frac{\partial}{\partial \theta}
$$

and the trapping operator

$$
-i \omega_{\xi}=-\frac{v}{2} \frac{\left(1-\xi^{2}\right)}{\mathcal{J}_{\psi} B} \frac{\partial \ln B}{\partial \theta} \frac{\partial}{\partial \xi} .
$$

The trapping operator appears when using the $(\xi, v)$ velocity-space coordinates, but is absent in GYRO and GS2 which use constant-of-motion coordinates. The trapped-particle regions correspond to $\xi^{2}<1-B(\theta) / B(\pi)$, as illustrated in Fig. 1. Next, we have the radial drift operator

$$
-i \omega_{\mathrm{d}}=\mathbf{v}_{D} \cdot \nabla_{\perp}
$$

where the perpendicular drift velocity (including the pressure-gradient drift) is

$$
\mathbf{v}_{D}=\frac{v^{2}}{2} \frac{\left(1+\xi^{2}\right)}{\Omega_{c a}} \frac{\mathbf{b} \times \nabla B}{B}+\frac{v^{2}}{2} \frac{\xi^{2}}{\Omega_{c a}} \frac{8 \pi}{B^{2}} \frac{d p}{d r}(\mathbf{b} \times \nabla r) .
$$


The pressure gradient drift produces an important stabilizing effect on ion-temperature-gradient (ITG) modes (otherwise known as Shafranov-shift stabilization) and should not be confused with the stabilizing effect of line-bending in the Ampère equation. The Poisson bracket has been used to simplify and combine the expressions for the gradient-drive and nonlinear terms, according to the convention

$$
[f, g] \doteq \frac{\partial f}{\partial r} \frac{\partial g}{\partial \alpha}-\frac{\partial g}{\partial r} \frac{\partial f}{\partial \alpha}
$$

Finally, on the right-hand side of Eq. (16), we have written the linearized gyrophase-averaged collision operator,

$$
C_{a b}^{\mathrm{GK}} \doteq \sum_{\mathbf{k}_{\perp}} \oint \frac{d \vartheta}{2 \pi} e^{i S(\mathbf{R})} e^{i \mathbf{k}_{\perp} \cdot \boldsymbol{\rho}_{a}} C_{a b}^{L}\left(f_{1 a, \mathbf{k}_{\perp}}, f_{1 b, \mathbf{k}_{\perp}}\right)
$$

The explicit form of the collision operator will be discussed in Sec. 2.5.

\subsection{Maxwell equations}

The gyrocenter Maxwell equations [19, 17] are written as

$$
\begin{aligned}
-\frac{1}{4 \pi} \nabla_{\perp}^{2} \delta \phi+\sum_{a} \frac{z_{a}^{2} e^{2}}{T_{a}} \int d^{3} v f_{0 a} \delta \phi & =\sum_{a} z_{a} e \int d^{3} v \mathcal{G}_{0 a} H_{a} \\
-\frac{1}{4 \pi} \nabla_{\perp}^{2} \delta A_{\|} & =\sum_{a} z_{a} e \int d^{3} v \frac{v_{\|}}{c} \mathcal{G}_{0 a} H_{a} \\
-\frac{1}{4 \pi} \delta B_{\|} & =\sum_{a} z_{a} e \int d^{3} v \frac{v_{\perp}^{2}}{\Omega_{c a} c} \mathcal{G}_{1 a} H_{a}
\end{aligned}
$$

where the operators $\mathcal{G}_{0 a}$ and $\mathcal{G}_{1 a}$ are defined in Eqs. (21) and (22).

\subsection{Collision operator}

Collision models with varying degree of sophistication are implemented in CGYRO. In the present work we describe only the most general form of the operator (the Sugama operator), and the Lorentz operator. The latter form is the one most suitable for intercode benchmarking. The Sugama operator [21] is a model for the complete linearized, gyroaveraged Fokker-Planck opertor that is designed to conserve particles, momentum and energy. It also satisfies adjointness relations and Boltzmann's H-theorem. We note that Abel et al. [22] have developed a similar operator for the special case of like-species collisions. The Sugama operator, importantly, extends the regime of validity to include the description of unlike-species collisions, including those with different temperatures $T_{a} \neq T_{b}$.

The general linearized collision operator is separated into a test particle component $C_{a b}^{T}$ and a field particle component $C_{a b}^{F}$, such that

$$
C_{a b}^{L}\left(H_{a, \mathbf{k}_{\perp}}, H_{b, \mathbf{k}_{\perp}}\right)=C_{a b}^{T}\left(H_{a, \mathbf{k}_{\perp}}\right)+C_{a b}^{F}\left(H_{b, \mathbf{k}_{\perp}}\right) .
$$

The test-particle part of the Sugama model [21] consists of the full linearized gyro-avergaged FokkerPlanck test-particle operator (pitch-angle scattering plus diffusion). This operator, including finite Larmor radius effects, is written as

$$
C_{a b}^{T}\left(H_{a, \mathbf{k}_{\perp}}\right)=C_{a b}^{T 0}\left(H_{a, \mathbf{k}_{\perp}}\right)-H_{a, \mathbf{k}_{\perp}} k_{\perp}^{2} \rho_{a}^{2} \frac{u_{a}^{2}}{2}\left[\nu_{a b}^{D}\left(1+\xi^{2}\right)+\nu_{a b}^{\|}\left(1-\xi^{2}\right)\right] .
$$


For the case of like-species and inter-ion species collisions (for which we assume $T_{a}=T_{b}$ ),

$$
C_{a b}^{T 0}\left(H_{a, \mathbf{k}_{\perp}}\right)=\nu_{a b}^{D} \mathcal{L} H_{a, \mathbf{k}_{\perp}}+\mathcal{D}\left(H_{a, \mathbf{k}_{\perp}}\right) .
$$

For the case of ion-electron and electron-ion collisions $\left(T_{i} \neq T_{e}\right)$, we neglect terms which are $\mathcal{O}\left(m_{e} / m_{i}\right)$ smaller than the dominant (electron) collision terms. This is a common approximation, and since $m_{e} / m_{D}<3 \times 10^{-4}$, the associated error is much smaller than a typical truncation error in the numerical discretization. Specifically,

$$
C_{i e}^{T 0}\left(H_{i, \mathbf{k}_{\perp}}\right)=0 \quad \text { and } \quad C_{e i}^{T 0}\left(H_{e, \mathbf{k}_{\perp}}\right)=\nu_{e i}^{D} \mathcal{L} H_{e, \mathbf{k}_{\perp}} .
$$

In Eqs. (36) and (37), $\mathcal{L}$ is the Lorentz (pitch-angle scattering) operator,

$$
\mathcal{L}=\frac{1}{2} \frac{\partial}{\partial \xi}\left(1-\xi^{2}\right) \frac{\partial}{\partial \xi}
$$

and $\mathcal{D}$ is the diffusion operator,

$$
\mathcal{D}_{a b}\left(H_{a, \mathbf{k}_{\perp}}\right)=\frac{1}{u_{a}^{2}} \frac{\partial}{\partial u_{a}}\left[\nu_{a b}^{\|}\left(\frac{1}{2} u_{a}^{4} \frac{\partial H_{a, \mathbf{k}_{\perp}}}{\partial u_{a}}+\frac{T_{a}}{T_{b}} u_{a}^{5} H_{a, \mathbf{k}_{\perp}}\right)\right],
$$

where the pitch-angle diffusion (deflection) rate $\nu^{D}$ is

$$
\nu_{a b}^{D}(v)=\frac{\tau_{a b}^{-1}}{u_{a}^{3}}\left[\frac{1}{\sqrt{\pi} u_{b}} e^{-u_{b}^{2}}+\operatorname{erf}\left(u_{b}\right)\left(1-\frac{1}{2 u_{b}^{2}}\right)\right],
$$

and the parallel velocity diffusion rate $\nu^{\|}$is

$$
\nu_{a b}^{\|}(v)=2 \frac{\tau_{a b}^{-1}}{u_{a}^{3}}\left[-\frac{1}{\sqrt{\pi} u_{b}} e^{-u_{b}^{2}}+\operatorname{erf}\left(u_{b}\right)\left(\frac{1}{2 u_{b}^{2}}\right)\right] .
$$

The field particle component is ad hoc, designed to conserve momentum and energy.

$$
C_{a b}^{F}\left(H_{b, \mathbf{k}_{\perp}}\right)=R_{a b}^{\mathrm{mom}}+R_{a b}^{\mathrm{ene}}
$$

where

$$
\begin{aligned}
R_{a b}^{\mathrm{mom}}=\eta_{a b}^{\mathrm{mom}}[ & J_{0}\left(\gamma_{a}\right) \int d^{3} v J_{0}\left(\gamma_{b}\right) \frac{H_{b, \mathbf{k}_{\perp}}}{f_{0 b}} C_{b a}^{T 0}\left(v_{\|} f_{0 b}\right) \\
& \left.+J_{1}\left(\gamma_{a}\right) \frac{v_{\perp}}{v_{\|}} \int d^{3} v J_{1}\left(\gamma_{b}\right) \frac{v_{\perp}}{v_{\|}} \frac{H_{b, \mathbf{k}_{\perp}}}{f_{0 b}} C_{b a}^{T 0}\left(v_{\|} f_{0 b}\right)\right], \\
R_{a b}^{\mathrm{ene}}= & \eta_{a b}^{\text {ene }} J_{0}\left(\gamma_{a}\right) \int d^{3} v J_{0}\left(\gamma_{b}\right) \frac{H_{b, \mathbf{k}_{\perp}}}{f_{0 b}} C_{b a}^{T 0}\left(u_{b}^{2} f_{0 b}\right) .
\end{aligned}
$$

Above, we have defined

$$
\begin{aligned}
\eta_{a b}^{\mathrm{mom}} & \doteq-\frac{m_{b}}{m_{a}} \frac{C_{a b}^{T 0}\left(v_{\|} f_{0 a}\right)}{\int d^{3} v v_{\|} C_{a b}^{T 0}\left(v_{\|} f_{0 a}\right)} \\
\eta_{a b}^{\mathrm{ene}} & \doteq-\frac{T_{b}}{T_{a}} \frac{C_{a b}^{T 0}\left(u_{a}^{2} f_{0 a}\right)}{\int d^{3} v u_{a}^{2} C_{a b}^{T 0}\left(u_{a}^{2} f_{0 a}\right)}
\end{aligned}
$$


For comparison, we also define a simple Lorentz collision model, which is standard in most gyrokinetic codes, and retains only the essential pitch-angle scattering dynamics. The Lorentz model, written as

$$
\begin{aligned}
C_{e e}^{L} & =\nu_{e e}^{D} \mathcal{L} H_{e, \mathbf{k}_{\perp}}, \\
C_{e i}^{L} & =\frac{\tau_{e i}^{-1}}{u_{a}^{3}} \mathcal{L} H_{e, \mathbf{k}_{\perp}}, \\
C_{i i}^{L} & =C_{i e}^{L}=0,
\end{aligned}
$$

employs the small electron-to-ion mass ratio approximation; that is, the model ignores terms of $\mathcal{O}\left(m_{e} / m_{i}\right)^{1 / 2}$ and smaller. Further, because it neglects the field particle component for all species, the Lorentz model is not momentum conserving. Despite its simplicity, simulation results show that the model is nevertheless relatively accurate for core plasmas. The physical explanation for the accuracy is related to the narrow boundary layer at the electron TP boundary. In the limit $\bar{\nu}_{e} \rightarrow 0$, the collision operator is only important in the TP boundary layer, with the contribution asymptotically dominated by the term $\partial^{2} \widetilde{H}_{a} / \partial \xi^{2}[23]$.

\section{Description of numerical discretization}

\subsection{Perpendicular spatial discretization: $(r, \alpha)$}

In what follows we will write the gyrokinetic and Maxwell equations in terms of the discrete indices $(p, n)$ which are the Fourier wavenumbers corresponding to the $(r, \alpha)$ coordinates. In other

words, we define the explicit form of the $2 \mathrm{D}$ wavenumber $\mathbf{k}_{\perp}$. We remark that for the system to admit periodic solutions of this type, we are assuming the maximal gyrokinetic ordering (often called flux-tube) for which the perpendicular eddy size is much smaller than the equilibrium scale length; that is, $\mathbf{k}_{\perp} a \gg 1$.

Introducing a periodic radial variable $x \doteq 2 \pi\left(r-r_{0}\right) / L \in[0,2 \pi)$, where $L$ is the radial domain size, we can approximate a real function $f(x, \alpha)$ as a finite two-dimensional Fourier series

$$
f(x, \alpha)=\sum_{n=-N_{\alpha}}^{N_{\alpha}} \sum_{p=-M}^{M} \tilde{f}(n, p) e^{i p x} e^{-i n \alpha},
$$

where $N_{r}=2 M$ is the total number of radial $(x)$ wavenumbers. Since we wish to use an even number of modes in the $x$-direction, we set $\tilde{f}(n \geq 0, M)=0$. Furthermore, because reality of physical fields implies

$$
\widetilde{f}(-n,-p)=\widetilde{f}^{*}(n, p),
$$

it is only necessary to evolve the gyrokinetic equation for $n \geq 0$, with negative values obtained by complex conjugation. For zonal modes $(n=0)$, we have the explicit restrictions

$$
\widetilde{f}(0, \pm M)=0 \text { and } \tilde{f}(0,0)=0,
$$

which must be carefully enforced during simulation. We expand the normalized distributions and fields according to

$$
\begin{aligned}
\frac{h_{a}}{f_{0 a}} & =\sum_{n=-N_{\alpha}}^{N_{\alpha}} \sum_{p=-M}^{M} \widetilde{h}_{a}(n, p) e^{i p x} e^{-i n \alpha} \\
\frac{e \Psi_{a}}{T_{e}} & =\sum_{n=-N_{\alpha}}^{N_{\alpha}} \sum_{p=-M}^{M} \widetilde{\Psi}_{a}(n, p) e^{i p x} e^{-i n \alpha} .
\end{aligned}
$$


For clarity, we remind the reader that $x, \alpha \in[0,2 \pi)$. With this representation, the eikonal phase of Eq. (18) is $S=p x-n \alpha$. Introducing unit vectors $\mathbf{e}_{x} \doteq \nabla r /|\nabla r|$ and $\mathbf{e}_{y} \doteq \mathbf{b} \times \mathbf{e}_{x}$, we have the corresponding wavenumbers

$$
\begin{aligned}
& k_{x}=\mathbf{e}_{x} \cdot \mathbf{k}_{\perp}=2 \pi p \frac{|\nabla r|}{L}-n \frac{\nabla r \cdot \nabla \nu}{|\nabla r|}, \\
& k_{y}=\mathbf{e}_{y} \cdot \mathbf{k}_{\perp}=\frac{n B}{|\nabla \psi|},
\end{aligned}
$$

where $k_{\perp}^{2}=k_{x}^{2}+k_{y}^{2}$. It is worth remarking that in the limit of large-aspect-ratio, unshifted circular equilibrium, we have $k_{x}=2 \pi p / L+s \theta k_{\theta}$ and $k_{y}=k_{\theta}$, where $k_{\theta}=n q / r$ and $s=(r / q) \partial q / \partial r$. Using Eqs. (52) through (55), it is trivial to write the field equations in spectral form. The spectral representation of the Poisson bracket is a generalized convolution

$$
(\widetilde{f} * \widetilde{g})(n, p) \doteq \sum_{p^{\prime}} \sum_{n^{\prime}} \widetilde{f}\left(n^{\prime \prime}, p^{\prime \prime}\right) \widetilde{g}\left(n^{\prime}, p^{\prime}\right)\left(n^{\prime \prime} p^{\prime}-n^{\prime} p^{\prime \prime}\right)
$$

where the sums are suitably restricted to acceptable values of the indices; that is, $n^{\prime}+n^{\prime \prime}=n$ and $p^{\prime}+p^{\prime \prime}=p$. To evolve the nonlinear gyrokinetic equations, the Poisson bracket must be treated carefully. It is well-known that for stable time-integration, dealiasing is required. This is explained in more detail in Sec. 3.8. The final dimensionless equation is

$$
\frac{\partial \widetilde{h}_{a}}{\partial \tau}-i\left(\Omega_{\theta}+\Omega_{\xi}+\Omega_{\mathrm{d}}\right) \widetilde{H}_{a}-i \Omega_{*} \widetilde{\Psi}_{a}+\frac{2 \pi a}{L} \frac{q \rho_{s}}{r} \widetilde{h}_{a} * \widetilde{\Psi}_{a}=\frac{a}{c_{s}} \sum_{b} C_{a b}^{L}\left(\widetilde{H}_{a}, \widetilde{H}_{b}\right)
$$

with $\tau \doteq\left(c_{s} / a\right) t$ the normalized time. In Sec. 3.5 we will describe an additional spectral dissipation term that can be added to the conservative approach in this section. In Eq. (57), the dimensionless streaming and trapping operators are

$$
\begin{aligned}
-i \Omega_{\theta} & =\frac{v_{\|}}{w_{s}} \frac{\partial}{\partial \theta}, \\
-i \Omega_{\xi} & =\frac{v_{t a}}{w_{s}} \frac{u_{a}}{\sqrt{2}}\left(1-\xi^{2}\right) \frac{\partial \ln B}{\partial \theta} \frac{\partial}{\partial \xi} .
\end{aligned}
$$

Above, we have introduced an effective velocity $w_{s}=c_{s}\left(\mathcal{J}_{\psi} B\right) / a$. In a plasma with general crosssectional shape, the drift operator is complicated and cannot be written in terms of simple trigonometric functions. We write it as

$$
-i \Omega_{\mathrm{d}}=a \frac{v_{t a}}{c_{s}} u_{a}^{2}\left[\left(1+\xi^{2}\right) \frac{\mathbf{b} \times \nabla B}{B}-\xi^{2} \beta^{*} \frac{B_{\mathrm{unit}}^{2}}{B^{2}} \mathbf{b} \times \nabla r\right] \cdot i \mathbf{k}_{\perp} \rho_{a},
$$

where $\beta^{*}$ is defined in Eq. (11). Finally, the gradient drive is represented by

$$
-i \Omega_{*}=\left[\frac{a}{L_{n a}}+\frac{a}{L_{T a}}\left(u_{a}^{2}-\frac{3}{2}\right)\right] i k_{\theta} \rho_{s} .
$$

We also have

$$
\widetilde{H}_{a}=\widetilde{h}_{a}+z_{a} \frac{T_{e}}{T_{a}} \widetilde{\Psi}_{a}
$$

where

$$
\widetilde{\Psi}_{a} \doteq J_{0}\left(\gamma_{a}\right)\left(\delta \widetilde{\phi}-\frac{v_{\|}}{c_{s}} \delta \widetilde{A_{\|}}\right)+\frac{\rho_{a} v_{t a}}{\rho_{s} c_{s}} \frac{v_{\perp}^{2}}{v_{t a}^{2}} \frac{J_{1}\left(\gamma_{a}\right)}{\gamma_{a}} \delta \widetilde{B_{\|}}
$$


and

$$
\begin{aligned}
v_{\|} & =\sqrt{2} \xi u_{a} v_{t a}, \\
v_{\perp}^{2} & =2 u_{a}^{2}\left(1-\xi^{2}\right) v_{t a}^{2}, \\
\gamma_{a} & =\sqrt{2} u_{a} \sqrt{1-\xi^{2}} k_{\perp} \rho_{a} .
\end{aligned}
$$

\subsection{Spectral form of the Maxwell equations}

The dimensionless, spectral Maxwell equations are

$$
\begin{aligned}
k_{\perp}^{2} \lambda_{D}^{2} \delta \widetilde{\phi}+\sum_{a} z_{a}^{2} \frac{T_{e}}{T_{a}} \int d^{3} v \frac{f_{0 a}}{n_{e}} \delta \widetilde{\phi} & =\sum_{a} z_{a} \int d^{3} v \frac{f_{0 a}}{n_{e}} J_{0}\left(\gamma_{a}\right) \widetilde{H}_{a}, \\
\frac{2}{\beta_{e, \text { unit }}} k_{\perp}^{2} \rho_{s}^{2} \delta \widetilde{A_{\|}} & =\sum_{a} z_{a} \int d^{3} v \frac{f_{0 a}}{n_{e}} \frac{v_{\|}}{c_{s}} J_{0}\left(\gamma_{a}\right) \widetilde{H}_{a}, \\
-\frac{2}{\beta_{e, \text { unit }}} \frac{B}{B_{\text {unit }}} \delta \widetilde{B_{\|}} & =\sum_{a} \int d^{3} v \frac{f_{0 a}}{n_{e}} \frac{m_{a} v_{\perp}^{2}}{T_{e}} \frac{J_{1}\left(\gamma_{a}\right)}{\gamma_{a}} \widetilde{H}_{a} .
\end{aligned}
$$

In the Poisson equation we have introduced the Debye length

$$
\lambda_{D} \doteq \sqrt{\frac{T_{e}}{4 \pi n_{e} e^{2}}}
$$

which is typically negligible unless $k_{\perp} \rho_{s} \gg 1$. Also, in these equations, the velocity integral is written as

$$
\int d^{3} v \frac{f_{0 a}}{n_{e}}=\frac{n_{a}}{n_{e}} \frac{2}{\sqrt{\pi}} \int_{0}^{\infty} d u_{a} u_{a}^{2} e^{-u_{a}^{2}} \int_{-1}^{1} d \xi
$$

\section{3. $\theta$-periodicity in the spectral representation}

The $\theta$-periodicity of real-space functions requires that

$$
\sum_{n, p} e^{i p x} e^{-i n \varphi-i n \nu(r, \theta)} \tilde{f}(n, p, \theta)=\sum_{n, p} e^{i p x} e^{-i n \varphi-i n \nu(r, \theta+2 \pi)} \tilde{f}(n, p, \theta+2 \pi) .
$$

However, noting that (see Ref. [15])

$$
\nu(r, \theta+2 \pi)-\nu(r, \theta)=-2 \pi q(r),
$$

we find that for each toroidal harmonic $n$,

$$
\sum_{p} e^{i p x} \tilde{f}(n, p, \theta)=\sum_{p} e^{i p x} e^{2 \pi i n q(r)} \tilde{f}(n, p, \theta+2 \pi) .
$$

Now, we quantize the box length via $L=n_{0} L_{0}$ where $L_{0}=r_{0} /(q s)$ is the fundamental balloning mode box length, and $n_{0}$ is the box multiplier. Note that $n_{0}$ measures the discrete number of different radial quantum numbers (typically denoted by the symbol $\theta_{0}$ ) that are allowed in the periodic domain of length $L$. Taylor expanding the safety factor according to

$$
\begin{aligned}
2 \pi i n q(r) & \simeq 2 \pi i n q_{0}+2 \pi i n \frac{q s}{r_{0}}\left(r-r_{0}\right), \\
& =2 \pi i n q_{0}+i n \frac{q s}{r_{0}} L x
\end{aligned}
$$


gives the condition

$$
\sum_{p} e^{i p x} \widetilde{f}(n, p, \theta) \sim e^{2 \pi i n q_{0}} \sum_{p} e^{i\left(p+n n_{0}\right) x} \widetilde{f}(n, p, \theta+2 \pi)\left[1+\mathcal{O}\left(\frac{\rho_{s}}{a}\right)\right] .
$$

Retaining only the first term in the Taylor expansion is thus consistent with the gyrokinetic ordering. Projection by continuous integration over the domain $x \in(0, L]$ gives

$$
\widetilde{f}(n, p, \theta)=e^{2 \pi i n q_{0}} \tilde{f}\left(n, p-n n_{0}, \theta+2 \pi\right) .
$$

This form of the periodicity condition gives what are effectively zero boundary conditions at $\widetilde{f}(n,-M, 0)$ and $\widetilde{f}(n, M-1,2 \pi)$ for $n>0$. Alternatively, we can introduce the discrete Fourier transform (DFT) pair:

$$
\begin{aligned}
F(n, j, \theta) & =\sum_{p=-M}^{M-1} e^{i \pi p j / M} \widetilde{f}(n, p, \theta), \\
\widetilde{f}(n, p, \theta) & =\frac{1}{2 M} \sum_{j=-M}^{M-1} e^{-i \pi p j / M} F(n, j, \theta) .
\end{aligned}
$$

In these expressions, the index $j$ labels the pesudospectral (real-space) radial gridpoints. Now, making the index $p$ cyclic with period $2 M$, gives what are effectively quasi-periodic boundary conditions at $\widetilde{f}(n,-M, 0)$ and $\widetilde{f}(n, M-1,2 \pi)$ for $n>0$.

\subsection{Poloidal discretization: $\theta$}

In the $\theta$-direction, the relevant piece of the gyrokinetic equation is associated with parallel motion and looks superficially like an advection

$$
\frac{\partial \widetilde{h}_{a}}{\partial \tau}+\frac{v_{\|}}{w_{s}} \frac{\partial \widetilde{H}_{a}}{\partial \theta} \quad \text { where } \quad \widetilde{H}_{a}=\widetilde{h}_{a}+z_{a} \frac{T_{e}}{T_{a}} \widetilde{\Psi}_{a}
$$

Because these terms will be integrated explicitly (see Sec. 3.9), we must focus on schemes that are suitable for explicit advection. In reality, because of the appearance of the field potential $\widetilde{\Psi}_{a}$, the parallel motion is more general than a pure advection so the standard approach to adding upwindtype dissipation must be modified to suit the more complicated form. Because Eq. (81) appears to advect $\widetilde{h}_{a}$, the natural starting point is to apply dissipation to $\widetilde{h}_{a}$. We remind the reader that explicit advection must always include grid-scale dissipation (i.e., upwind) for stability [24]. While dissipation of $\widetilde{h}_{a}$ is effective for electrostatic simulation, it performs poorly at moderate to high $\beta_{e, \text { unit. }}$. Indeed, experience shows that dissipating

$$
\widetilde{g}_{a} \doteq \widetilde{h}_{a}-z_{a} \frac{v_{\|}}{c_{s}} \delta \widetilde{A_{\|}}
$$

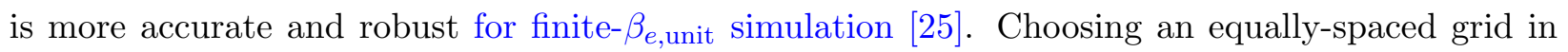
the poloidal angle $\left\{\theta_{j}\right\}$, we use difference formulae for equally-spaced grids to arrive at the scheme

$$
\frac{\partial\left(\widetilde{h}_{a}\right)_{j}}{\partial \tau}+\frac{v_{\|}}{w_{s}} D^{(n)}\left(\widetilde{H}_{a}\right)_{j}+\left|\frac{v_{\|}}{w_{s}}\right| S^{(n)}\left(\widetilde{g}_{a}\right)_{j} .
$$

Above, $D^{n}$ is the $(n+1)$-point finite-difference formula for the 1st derivative, and $S^{n}$ is the $(n+1)$ point finite-difference formula for the $n^{\text {th }}$ derivative multiplied by the standard weighting factor for 
upwind dissipation [24]. For example, if $n=6$, we have the following stencils that combine to give the $5^{\text {th }}$-order upwind scheme

$$
\begin{aligned}
D^{(6)} f_{j} & =\frac{+f_{j+3}-9 f_{j+2}+45 f_{j+1}+0 f_{j}-45 f_{j-1}+9 f_{j-2}-f_{j-3}}{60 \Delta \theta} \\
S^{(6)} f_{j} & =\frac{-f_{j+3}+6 f_{j+2}-15 f_{j+1}+20 f_{j}-15 f_{j-1}+6 f_{j-2}-f_{j-3}}{60 \Delta \theta}
\end{aligned}
$$

where $\Delta \theta \doteq \theta_{j}-\theta_{j-1}$. The role of $S$ as a grid-scale dissipation is clear by noting that

$$
S^{(6)} \sim-\frac{(\Delta \theta)^{5}}{60} \frac{\partial^{6} f}{\partial \theta^{6}}
$$

so that the overall advection scheme combines a $6^{\text {th }}$-order centered difference with a $6^{\text {th }}$-order filter to suppress grid-scale oscillation. These formulae illustrate a key feature of upwind dissipation; namely, that the strength of the dissipation vanishes as the grid is refined, so that the continuum limit of the equations is obtained naturally as the number of gridpoints increases. A more explicit estimate of the numerical damping rate due to the numerical dissipation is simple to derive. We assume the mode has a Gaussian envelope $\exp \left[-\lambda(\theta / \pi)^{2}\right]$, where $\lambda$ is the effective eigenmode width. This form is typical of ballooning eigenmodes that are well-localized, such that the maximum numerical dissipation rate is

$$
\gamma_{\text {upwind }} \simeq 20 \frac{a}{q R} \frac{v}{c_{s}} \frac{\lambda^{3}}{N_{\theta}^{5}} .
$$

While the upwind scheme in Eq. (83) performs well electromagnetically for $k_{\perp} \rho_{s}>0.1$, the dissipation can cause some inaccuracy related to violation of number conservation for $k_{\perp} \rho_{s}<$

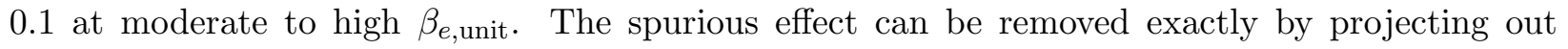
the gyrocenter density perturbation caused by the dissipation. The resulting conservative upwind scheme takes the form

$$
\frac{\partial\left(\widetilde{h}_{a}\right)_{j}}{\partial \tau}+\frac{v_{\|}}{w_{s}} D^{(n)}\left(\widetilde{H}_{a}\right)_{j}+\left|\frac{1}{w_{s}}\right| S^{(n)}\left[\left|v_{\|}\right|\left(\widetilde{g}_{a}\right)_{j}-\frac{J_{0}\left(\gamma_{a}\right) \int d^{3} v f_{0 a} J_{0}\left(\gamma_{a}\right)\left|v_{\|}\right|\left(\widetilde{g}_{a}\right)_{j}}{\int d^{3} v f_{0 a} J_{0}\left(\gamma_{a}\right)^{2}}\right]
$$

By conservative, we mean conservation of gyrocenter number with respect to the numerical dissipation. Thus the dissipation adds no gyrocenter density perturbation. To our knowledge this conservative upwind scheme has not been previously reported, but it appears to yield very accurate discretization in the long-wavelength, high- $\beta$ limit where the well-known Ampère cancellation problem [7] arises. In addition, performance is also improved in the high- $k_{\perp}$ regime associate with ETG modes.

\subsection{Spectral upwind diffusion for radial drift operator}

It is important to add some dissipation to the radial drift motion in order to enforce an effective outgoing-wave boundary condition for ballooning modes. This dissipation is also required to smooth over sub-grid-scale numerical disturbances in cases for which the radial grid spacing is much larger than an electron gyroradius [7]. While simulations can be done in the absence of this dissipation, the convergence properties of the algorithm are greatly improved when dissipation is added. The approach is to replace the spectral radial derivative with a discrete approximation that adds a small 
numerical disspation that is the Fourier-space equivalent of the centered-difference dissipation in real space. First, we write the original radial drift term in Eq. (57) without dissipation as

$$
\begin{aligned}
-i \Omega_{\mathrm{r}} \widetilde{H}_{a}= & a \frac{v_{t a}}{c_{s}} u_{a}^{2}\left(1+\xi^{2}\right)\left(\frac{\mathbf{b} \times \nabla B}{B} \cdot \nabla r\right) 2 \pi i p \frac{\rho_{a}}{L} \widetilde{H}_{a} \\
& \doteq \hat{\Omega}_{r}(2 \pi i p) \widetilde{H}_{a}
\end{aligned}
$$

Then, the spectral upwind formula can be implemented using the substitution

$$
-i \Omega_{r} \widetilde{H}_{a} \rightarrow \hat{\Omega}_{r}(2 \pi i p) \widetilde{H}_{a}+\left|\hat{\Omega}_{r}\right| N_{r} \mathcal{P}^{(n)}(\zeta) \widetilde{h}_{a},
$$

where $\zeta \doteq 2 \pi p / N_{r} \in[-\pi, \pi)$, and the form of $\mathcal{P}^{(n)}(\zeta)$ depends on the desired order of dissipation. The formulae for orders 2 through 8 are

$$
\begin{aligned}
\mathcal{P}^{(2)}(\zeta) & =1-\cos (\zeta) \\
\mathcal{P}^{(4)}(\zeta) & =\frac{3-4 \cos (\zeta)+\cos (2 \zeta)}{6} \\
\mathcal{P}^{(4)}(\zeta) & =\frac{20-30 \cos (\zeta)+12 \cos (2 \zeta)-2 \cos (3 \zeta)}{60} \\
\mathcal{P}^{(8)}(\zeta) & =\frac{70-112 \cos (\zeta)+56 \cos (2 \zeta)-16 \cos (3 \zeta)+2 \cos (4 \zeta)}{280}
\end{aligned}
$$

We are not aware of this spectral equivalent of upwind dissipation being previously reported in the literature, but it is a straightforward analog to the real-space formulation used with success in GYRO [7].

\subsection{Pitch-angle discretization: $\xi$}

We have three types of operations in pitch angle: integration (required for velocity integration), first-derivative (particle trapping) and the Lorentz operator (collisions):

$$
\int_{-1}^{1} d \xi, \quad \frac{\partial}{\partial \xi} \quad \text { and } \quad \mathcal{L}=\frac{\partial}{\partial \xi}\left(1-\xi^{2}\right) \frac{\partial}{\partial \xi} .
$$

The treatment of the velocity-space pitch-angle by expansion in a finite Legendre series is standard and has a long history in plasma physics. The continuous representation of functions is taken to be

$$
f(\xi) \sim \sum_{k=0}^{n-1} c_{k} P_{k}(\xi)
$$

where in this section we abbreviate $N_{\xi}=n$, with $N_{\xi}$ the number of pseudospectral mesh points. The polynomials $P_{k}$ satisfy the orthogonality condition

$$
\int_{-1}^{1} d \xi P_{k}(\xi) P_{\ell}(\xi)=\frac{1}{k+1 / 2} \delta_{k \ell}
$$

where $P_{k}$ has $k$ real roots,

$$
-1<\xi_{1}<\xi_{2}<\cdots<\xi_{k}<1 .
$$

Explicit forms for the polynomials, as well as the recursion relations to generate them, are wellknown and we do not repeat them here. The integration nodes for the $n^{\text {th }}$ order scheme are given 
by the roots $\left\{\xi_{i}\right\}_{1}^{n}$ of $P_{n}$, and the integration weights $\left\{w_{i}^{(\xi)}\right\}_{1}^{n}$ are computed from the standard formula

$$
w_{i}^{(\xi)}=\frac{2}{\left(1-\xi_{i}^{2}\right)\left[P_{n}^{\prime}\left(\xi_{i}\right)\right]^{2}} .
$$

The associated Gauss-Legendre integration rule is

$$
\int_{-1}^{1} d \xi f(\xi) \sim \sum_{i=1}^{n} w_{i}^{(\xi)} f\left(\xi_{i}\right) .
$$

The most straightforward method to determine the roots, $\xi_{i}$, is via direct root-finding. This works well for the Legendre polynomials but in the more general case there are more sophisticated methods such as the Golub-Welsch (GW) algorithm [26]. Construction of pseudospectral matrix representations for the derivative and Lorentz operators is now straightforward. Operating on the continuous representation, Eq. (97), with $\mathcal{L}$ and $\partial / \partial \xi$ and then evaluating each expression at the Gauss-Legendre nodes gives

$$
\begin{aligned}
\left(\frac{\partial}{\partial \xi}\right)_{i j} & =d_{i k}\left(m^{-1}\right)_{k j} \\
(\mathcal{L})_{i j} & =l_{i k}\left(m^{-1}\right)_{k j}
\end{aligned}
$$

where

$$
d_{i j}=P_{j}^{\prime}\left(\xi_{i}\right), \quad l_{i j}=-j(j-1) P_{j}\left(\xi_{i}\right) \quad \text { and } \quad m_{i j}=P_{j}\left(\xi_{i}\right) .
$$

Because high-order polynomials are strongly dissipated by collisions, we expect this discretization to be particularly accurate in the limit of strong collisions. A powerful feature of the Legendre series is to enforce the correct boundary condition at $\xi= \pm 1$; namely, regularity. This is in contrast to discretization of the pitch-angle scattering operator using finite-difference formulae, for which unphysical zero-derivative boundary conditions or one-sided differences are sometimes used for simplicity.

\subsection{Energy discretization: $u_{a}$}

In a collisionless gyrokinetic simulation (or more generally for collisions in the absence of energy diffusion) derivatives in energy are not required. Thus only a quadrature rule for the energy integration is required to implement the energy discretization. This is the case in GYRO. However, energy diffusion makes it necessary to design a discretization scheme for which spectrally accurate integrals and derivatives can be evaluated without pathologies due to endpoints - for example $\partial / \partial u_{a}$ as $u_{a} \rightarrow 0$. Moreover, because the energy domain is semi-infinite, standard finite-domain pseudospectral methods (i.e., Gauss-Legendre) are not directly applicable, and Laguerre pseudospectral methods are known to perform poorly. To bridge this gap, we have developed a new pseudospectral method based on a set of nonstandard orthogonal polynomials [27]. The critical feature is that the energy domain is truncated so that the maximum energy node can be controlled independent of the number of quadrature nodes.

We introduce the polynomials $Q_{k}$, defined to be orthogonal over the finite interval $u \in[0, b]$ with Maxwellian weight:

$$
\int_{0}^{b} d u e^{-u^{2}} Q_{k}(u) Q_{\ell}(u)=\gamma_{k} \delta_{k \ell}
$$

$Q_{k}(u)$ has $k$ real roots

$$
0<u_{1}<u_{2}<\cdots<u_{k}<b
$$


In the limit $b \rightarrow 0$, the polynomials are just shifted monic Legendre polynomials, whereas for $b \rightarrow \infty$ we recover half-range Hermite polynomials [27, 28]. The first few (monic) polynomials can be computed analytically using the Gram-Schmidt process. For example,

$$
\begin{aligned}
& Q_{0}(u)=1, \\
& Q_{1}(u)=u-\frac{1-e^{-b^{2}}}{\sqrt{\pi} \operatorname{erf}(b)} .
\end{aligned}
$$

For the higher-order polynomials it is preferrable to compute them for a given value of $u$ from the associated recursion relation

$$
Q_{k+1}(u)=\left(u+\alpha_{k}\right) Q_{k}+\beta_{k} Q_{k-1},
$$

with

$$
\begin{aligned}
\alpha_{k} & =-\frac{1}{\gamma_{k}} \int_{0}^{b} d u e^{-u^{2}} u Q_{k}^{2}(u), \\
\beta_{k} & =-\frac{\gamma_{k}}{\gamma_{k-1}} .
\end{aligned}
$$

The first and second derivatives of $Q_{k}$ can also be computed recursively using

$$
\begin{aligned}
& Q_{k+1}^{\prime}(u)=\left(u+\alpha_{k}\right) Q_{k}^{\prime}(u)+\beta_{k} Q_{k-1}^{\prime}(u)+Q_{k}(u), \\
& Q_{k+1}^{\prime \prime}(u)=\left(u+\alpha_{k}\right) Q_{k}^{\prime \prime}(u)+\beta_{k} Q_{k-1}^{\prime \prime}(u)+2 Q_{k}^{\prime}(u) .
\end{aligned}
$$

Steen [27] has derived simple recursive formulas for the recursion coefficients

$$
\begin{aligned}
\gamma_{k} & =\frac{k}{2} \gamma_{k-1}-\frac{1}{2}\left[e^{-u^{2}} Q_{k}(u) Q_{k-1}(u)\right]_{0}^{b}, \\
\alpha_{k} & =\frac{1}{2 \gamma_{k}}\left[e^{-u^{2}} Q_{k}^{2}(u)\right]_{0}^{b} .
\end{aligned}
$$

with $\beta_{k}$ given by Eq. (111). The integration nodes for the $n^{\text {th }}$ order scheme are given by the roots $\left\{u_{i}\right\}_{1}^{n}$ of $Q_{n}$, and the integration weights $\left\{w_{i}^{(u)}\right\}_{1}^{n}$ are computed from the standard formula

$$
w_{i}^{(u)}=\frac{\gamma_{n-1}}{Q_{n}^{\prime}\left(u_{i}\right) Q_{n-1}\left(u_{i}\right)}
$$

which follows from the Christoffel-Darboux identity. The associated discrete integration rule is

$$
\int_{0}^{b} d u e^{-u^{2}} f(u) \sim \sum_{i=1}^{n} w_{i}^{(u)} f\left(u_{i}\right) .
$$

An important point, which has a fundamental impact on the numerical approach to evaluating the nodes and weights, is that the recursive formulae above are unstable. This means that despite being computationaly efficient, our choice to use the method of Steen does not eliminate the well-known problem of progressive ill-conditioning of the classical procedure to contruct the nodes and weights [29]. A simple solution to the problem, which does not require use of more advanced analytic methods $[30,26]$, is to evaluate Steen's formulae, and then carry out the subsequent root-finding using the Python arbitrary-precision mpmath [31] library. Using 50 decimal digits of precision in the calculations yields integration nodes and weights accurate to the full 16 digits required for double 

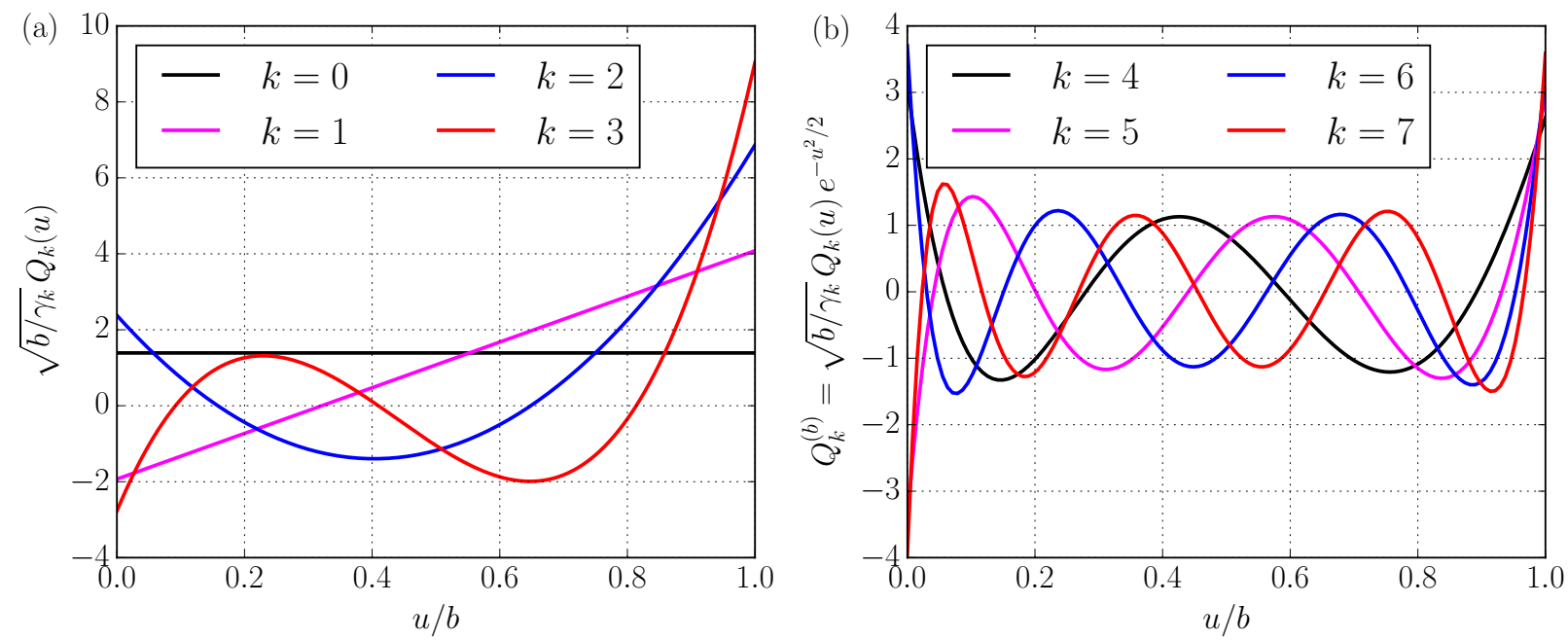

Figure 3: Orthogonal polynomials, $Q_{k}$, for $b^{2}=8$. Left plot (a) shows $k=0,1,2$, 3 , whereas right frame (b) shows $k=4,5,6,7$. Note the difference in overall normalization between (a) and (b).

precision gyrokinetic simulation. We also compute the first and second pseudospectral derivative matrices,

$$
\left(\frac{\partial}{\partial u}\right)_{i j} \text { and }\left(\frac{\partial^{2}}{\partial u^{2}}\right)_{i j}
$$

according to the same procedure as in Sec. 3.6. For simulation, we need to also define modified weights $\hat{w}^{(u)}$ more directly applicable to the gyrokinetic problem. These are

$$
\frac{4}{\sqrt{\pi}} \int_{0}^{b} d u u^{2} e^{-u^{2}} f(u)=\sum_{i=1}^{n} \hat{w}_{i}^{(u)} f\left(u_{i}\right) \quad \text { with } \quad \hat{w}_{i}^{(u)}=\frac{4 u_{i}^{2}}{\sqrt{\pi}} w_{i}^{(u)} .
$$

Although it is not necessary to define orthonormal polynomials for construction of the pseudospectral method, they are convenient for the purpose of plotting. To this end, we perform the change of variable $u=b \bar{u}$ to yield

$$
\int_{0}^{1} d \bar{u} Q_{k}^{(b)}(\bar{u})=1 \quad \text { where } \quad Q_{k}^{(b)}(\bar{u})=\sqrt{\frac{b}{\gamma_{k}}} e^{-b^{2} \bar{u}^{2} / 2} Q_{k}(b \bar{u}) .
$$

We briefly summarize the case $n=8, b^{2}=8$. First, plots of the interpolating polynomials for $0 \leq k \leq 7$ are shown in Fig. 3, with the roots of $Q_{8}$ illustrated in Fig. 4. Values for the integration nodes and modified weights are tabulated in Table 1. In the latter table we also give the value of $\nu_{e e}^{D}$ at the location of the node points, illustrating the transition from collisional to collisionless regimes as $i$ increases.

\subsection{Nonlinear discretization}

The treatment of the quadratic nonlinearity, through numerical evaluation of the convolution given in Eq. (56), is done in a standard way using a 2D Fast-Fourier transform (FFT) with dealiasing [32]. The convolution can be done directly, and exactly, by direct summation but to do so would be prohibitively slow for typical nonlinear simulations. Taking the FFT directly, multiplying the real-space function, and then returning to Fourier space via inverse FFT gives rise to aliasing. The 


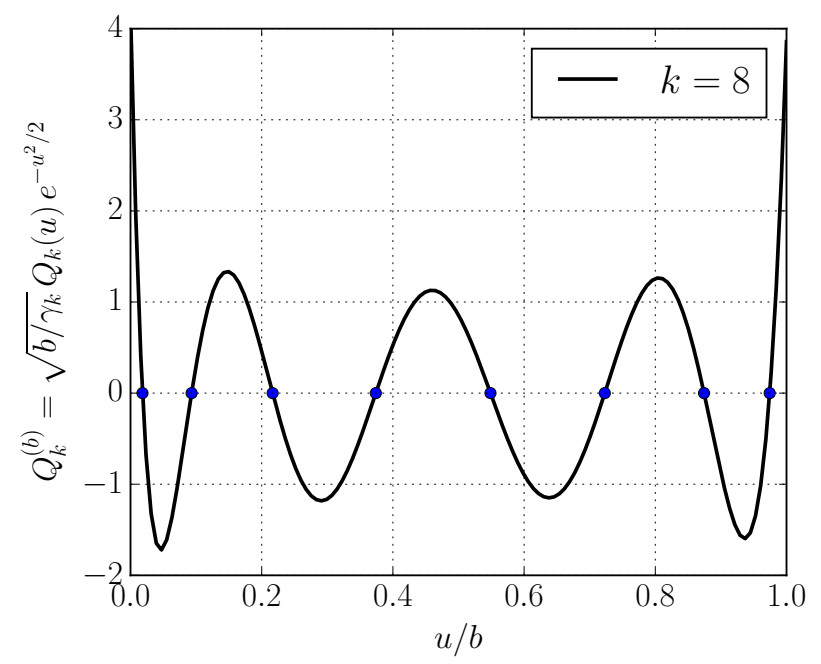

Figure 4: Polynomial $Q_{8}^{(b)}(w)$ for $b^{2}=8$ with zeros $u_{1}<u_{2}<\cdots<u_{8}$. The zeros of $Q_{8}$ are the pseudospectral nodes (or equivalently, the quadrature nodes) for the 8-point method.

Table 1: Summary of energy grid parameters for $N_{u}=8, b^{2}=8$ and $\bar{\nu}_{e}=1.0$.

\begin{tabular}{cccc}
\hline \hline$i$ & $u_{i}$ & $\hat{w}_{i}^{(u)}$ & $\left(a / c_{s}\right) \nu_{e e}^{D}(v)$ \\
\hline 1 & 0.04481 & 0.00052 & 374.415 \\
2 & 0.22722 & 0.02723 & 14.4215 \\
3 & 0.52603 & 0.16385 & 2.57666 \\
4 & 0.90922 & 0.33948 & 0.78257 \\
5 & 1.35004 & 0.30717 & 0.30578 \\
6 & 1.82579 & 0.13006 & 0.14010 \\
7 & 2.30486 & 0.02727 & 0.07400 \\
8 & 2.70338 & 0.00329 & 0.04715 \\
\hline \hline
\end{tabular}

standard approach to eliminate aliasing is to add to and then zero-pad the spectral representation by a factor of $3 / 2$, take the FFTs on the extended mesh, multiply the real-space product, then retain only the original wavenumbers. The dealiased convolution conserves a number of important invariants and thereby eliminates a class of nonlinear instabilities from the numerical solution. To perform the forward and inverse FFTs we use FFTW [33]. First, we perform a series of four 2D complex-to-real (c2r) transforms

$$
(i p) \tilde{f} \underset{\mathrm{c} 2 \mathrm{r}}{\longrightarrow} \frac{\partial f}{\partial x}, \quad(i p) \widetilde{g} \underset{\mathrm{c} 2 \mathrm{r}}{\longrightarrow} \frac{\partial g}{\partial x}, \quad(i n) \widetilde{f} \underset{\mathrm{c} 2 \mathrm{r}}{\longrightarrow} \frac{\partial f}{\partial y} \quad \text { and } \quad(i n) \widetilde{g} \underset{\mathrm{c} 2 \mathrm{r}}{\longrightarrow} \frac{\partial g}{\partial y},
$$

such that all arrays are extended and zero-padded by a factor of $3 / 2$. The real-space product is then taken, followed by the inverse transform of the entire nonlinearity via a single $2 \mathrm{D}$ real-to-complex (r2c) transform

$$
-\frac{\partial f}{\partial x} \frac{\partial g}{\partial y}+\frac{\partial g}{\partial x} \frac{\partial f}{\partial y} \underset{\mathrm{r} 2 \mathrm{c}}{\longrightarrow} \widetilde{f} * \widetilde{g}
$$

In the above we have set $y=-\alpha$ to keep the sign convention the same in both dimensions. Writing this expensive computational kernel completely in terms of FFTW is an important strategy to obtain 
good performance on current and future GPU and multicore computer systems.

\subsection{Time integration algorithm}

The time integration algorithm uses a mixed implicit-explicit approach, with nearly all the collisionless dynamics treated explicitly with a high-order multi-stage scheme, and the collisional dynamics done implicitly. We start by writing the gyrokinetic equation in the form

$$
\frac{\partial \widetilde{h}_{a}}{\partial \tau}+A\left(\widetilde{H}_{a}, \widetilde{\Psi}_{a}\right)+B\left(\widetilde{H}_{a}, \widetilde{\Psi}_{a}\right)=0
$$

where

$$
\begin{aligned}
& A\left(\widetilde{H}_{a}, \widetilde{\Psi}_{a}\right) \doteq-i\left(\Omega_{\theta}+\Omega_{\mathrm{d}}\right) \widetilde{H}_{a}-i \Omega_{*} \widetilde{\Psi}_{a}+\frac{2 \pi a}{L} \frac{q \rho_{s}}{r} \widetilde{h}_{a} * \widetilde{\Psi}_{a} \\
& B\left(\widetilde{H}_{a}, \widetilde{\Psi}_{a}\right) \doteq-i \Omega_{\xi} \widetilde{H}_{a}-\sum_{b} C_{a b}^{L}\left(\widetilde{H}_{a}, \widetilde{H}_{b}\right) .
\end{aligned}
$$

The explicit advance requires solution of the equation

$$
\frac{\partial \widetilde{h}_{a}}{\partial \tau}+A\left(\widetilde{H}_{a}, \widetilde{\Psi}_{a}\right)=0
$$

At every stage of the multi-stage scheme, an explicit expression for the partially-advanced $\widetilde{h}_{a}$ must be substituted into the Maxwell equations to obtain the partially-advanced fields and thus the partially-advanced $\widetilde{\Psi}_{a}$. For example, in the present work, we use RK4 to time-advance the explicit stage by an amount $\Delta \tau$. Since this advance contains fast linear and nonlinear timescales, we speculate that some performance benefit may be obtained by a more specialized or higher-order integrator. In general, the timestep restriction for the explicit advance is set by the oscillation frequency of the fastest Alfvén wave on the numerical mesh [25]. It is important to note that this can set a significantly more restrictive limit on the timestep, especially in the case of low $k_{\perp}$ and low $\beta_{e, \text { unit }}$, than the simple advective Courant condition for electron streaming. We remark that an explicit scheme was chosen to ensure maximum efficiency for nonlinear multiscale simulations (i.e., treating both electron and ion spatial scales simultaneously). This requires a very large number of radial $\left(N_{r}=2 M>1024\right)$ and binormal $\left(N_{\alpha}>256\right)$ wavenumbers. Attempting to use an implicit advection algorithm is useful for small linear case, but prohibitively expensive in terms of storage and speed, for multiscale cases.

Next, for the implicit advance, we solve

$$
\frac{\partial \widetilde{h}_{a}}{\partial \tau}+B\left(\widetilde{H}_{a}, \widetilde{\Psi}_{a}\right)=0
$$

The solution of this equation is subtle because the fields change during the implicit advance. To clarify this, we write the equation to be solved as

$$
\frac{\partial \widetilde{H}_{a}}{\partial \tau}-z_{a} \frac{T_{e}}{T_{a}} \frac{\partial \widetilde{\Psi}_{a}}{\partial \tau}-i \Omega_{\xi} \widetilde{H}_{a}=\sum_{b} C_{a b}^{L}\left(\widetilde{H}_{a}, \widetilde{H}_{b}\right)
$$

We note that in addition to the collision operator, the particle trapping term is also retained in the implicit advance. This is done because the pitch-angle derivative fits naturally into the formalism for evaluating the Lorentz part of the collision operator. Generally, the term is small, however, 
and we have verified that results are the same if it is treated in the explicit advance. To solve the equation using the Crank-Nicolson method, we first make the replacements

$$
\frac{\partial \widetilde{H}_{a}}{\partial \tau} \rightarrow \frac{\widetilde{H}_{a}^{+}-\widetilde{H}_{a}^{-}}{\Delta \tau} \text { and } \quad \widetilde{H}_{a} \rightarrow \frac{\widetilde{H}_{a}^{+}+\widetilde{H}_{a}^{-}}{2}
$$

where $\widetilde{H}_{a}^{-}$and $\widetilde{H}_{a}^{+}$are the initial and final distributions respectively. Using the Maxwell equations to express all fields in terms of velocity-space sums of $\widetilde{H}_{a}$, we arrive at a single matrix equation for all species. This matrix equation can be written in schematic form as

$$
\left[\begin{array}{c}
\widetilde{H}_{1}^{+} \\
\widetilde{H}_{2}^{+} \\
\vdots \\
\widetilde{H}_{N_{s}}^{+}
\end{array}\right]=\mathbb{M}\left[\begin{array}{c}
\widetilde{H}_{1}^{-} \\
\widetilde{H}_{2}^{-} \\
\vdots \\
\widetilde{H}_{N_{s}}^{-}
\end{array}\right],
$$

where $\operatorname{rank}(\mathbb{M})=N_{s} N_{\xi} N_{u}$. Because there are no fast physical timescales associated with the implicit advance, we see little benefit in increasing the accuracy beyond the 2nd-order CrankNicolson method. Because the accuracy of the explicit and implicit advances are both second-order or greater, the accuracy of the overall split scheme is also second order except for the first and last timesteps. This feature of operator splitting is well-known [24].

\section{Simulation and benchmark results}

For ion-temperature-gradient (ITG) test case parameters, we use the General Atomics (GA) standard case parameters [34]: $R_{0} / a=3, r / a=0.5, q=2, T_{i}=T_{e}, a / L_{n i}=a / L_{n e}=1, a / L_{T i}=a / L_{T e}=3$. The geometry is a local Grad-Shafranov unshifted circular equilibrium generated using the Miller formalism $[35,36]$. Other simulation parameters will be noted on a case-by-case basis.

\subsection{Convergence rate of numerical schemes}

To measure the rate of convergence in each dimension, we simulate unstable drift-waves at successively higher numerical resolution. To assess the order of convergence, we use a scheme that does not rely on having either an analytic or "high resolution" numerical solution from which to compute the absolute error. Instead, we use a simple and flexible approach to estimate the convergence rate based on successive approximations. Let $f_{N}$ be the approximation generated by a discretization scheme of order $n$ applied at $N$ mesh points. We know

$$
f_{N}=f_{\infty}+\alpha\left(\frac{\lambda}{N}\right)^{n}
$$

for some parameters $\alpha$ and $\lambda$. The difference between two successive approximations is

$$
\Delta f_{N} \doteq f_{N}-f_{N+1}=\alpha \lambda^{n}\left[\frac{1}{N^{n}}-\frac{1}{(N+1)^{n}}\right]
$$

This can be written as

$$
\ln \left(\Delta f_{N}\right) \sim \ln \left(\alpha n \lambda^{n}\right)-(n+1) \ln N+\mathcal{O}\left(\frac{1}{N}\right)
$$

which has negative slope with magnitude $(n+1)$ when plotted on a logarithmic $N$-axis. 

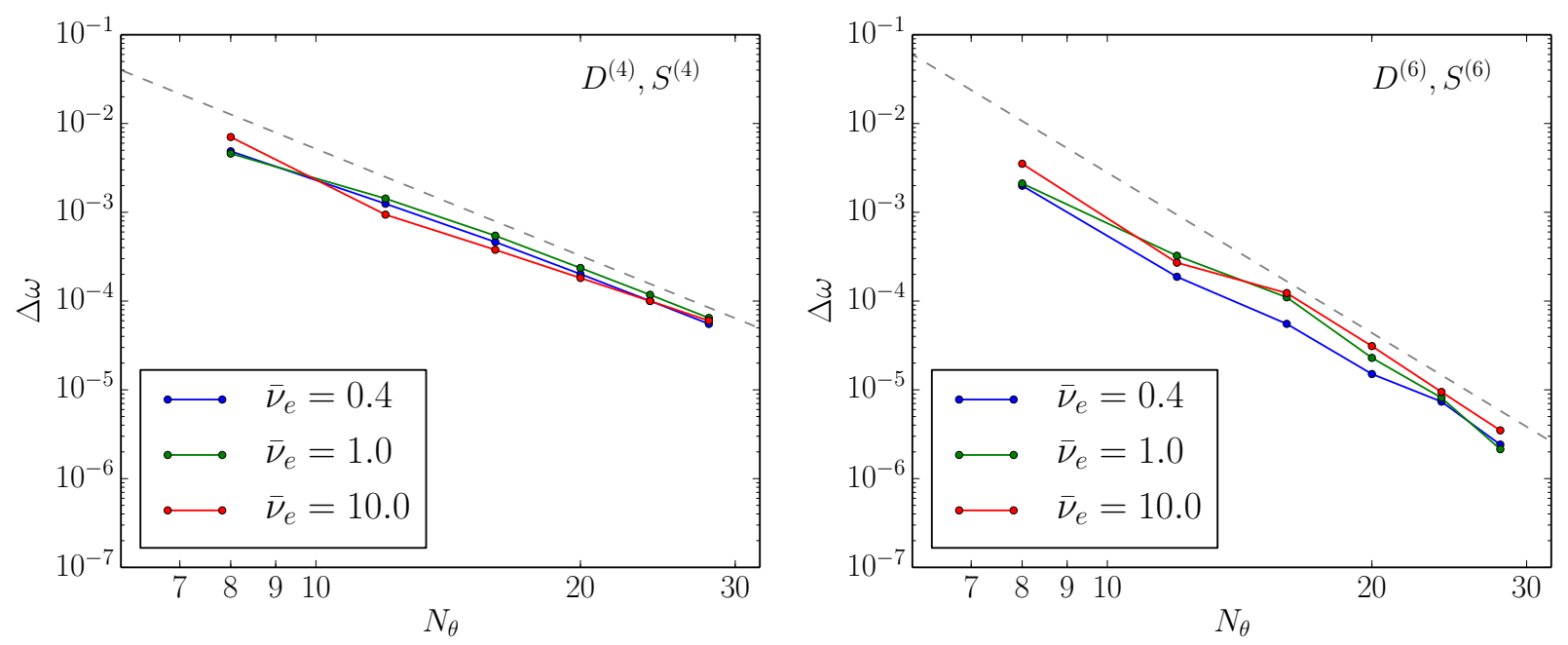

Figure 5: Convergence of the linear eigenmode frequency with respect to the number of meshpoints in $\theta$ for different values of the collision frequency $\bar{\nu}_{e}$. Left frame shows the $3^{\text {rd }}$-order upwind scheme, whereas right frame shows the $5^{\text {th }}$-order scheme as detailed in Sec. 3.4. The dashed lines illustrate $3^{\text {rd }}$ and $5^{\text {th }}$-order convergence rates, respectively. Note that for $N_{\theta}>16$, the maximal order of convergence is achieved.

Based on this approach, we carry out numerical convergence scans for a linear electrostatic $\left(\beta_{e, \text { unit }}=0\right)$ ITG eigenmode at $k_{\theta} \rho_{s}=0.3$. Results are shown in Figs. 5 and 6 . All scans were done using gyrokinetic electrons and ions, using the full Sugama collision operator. First, in Fig. 5, the theoretical order of convergence of the poloidal discretization, as described in Sec. 3.4, is demonstrated. In Fig. 6, spectral convergence (i.e., rate of convergence increasing with $N$ ) in both $N_{\xi}$ and $N_{u}$ is observed. Additionally, the absolute error in $\xi$ discretization decreases rapidly with increasing $\bar{\nu}_{e}$ due to the smoothing effect of pitch-angle diffusion in the vicinity of the trappedpassing boundary. Convergence in $u$, however, is roughly independent of the collisionality due to the lack of complex structure in energy in the collisionless limit.

\subsection{Energy-dependence of the collision operator}

It is perhaps not well-appreciated that for typical tokamak parameters, the gyrokinetic equation will exhibit highly-collisional behaviour at the lowest energy meshpoints, with a transition to essentially collisionless behaviour at the highest energies. The data in Table 1, together with corresponding plots of the distribution function in Fig. 7, illustrate this transition clearly. Note that the overall variation of $\nu_{e e}^{D}$ from the lowest to highest velocity is nearly a factor of $10^{5}$. Thus it is important for the numerical methods to be robust in the limit that the effective collision frequency is very large.

\subsection{Linear drift-wave and zonal flow benchmarks}

CGYRO has been successfully benchmarked with GYRO and GENE [11,3] for linear driftwave physics when both codes use a simple Lorentz pitch angle scattering model [see definition in Eqs. (46)-(48)]. Fig. 8 shows the results of this comparison for the standard case parameters at $k_{\theta} \rho_{s}=0.3$. It is well-known that trapped electrons provide a strong ITG drive mechanism [37], and thus electron collisions, by reducing the fraction of trapped electrons, reduce the ITG growth rate. All three codes verify this effect, and show good agreement in both the linear growth rate and real frequency over a broad range of collisionality. A comparison of Lorentz and Sugama operators in CGYRO for the same case, as shown in Fig. 9, shows that the differences in operators are negligible 

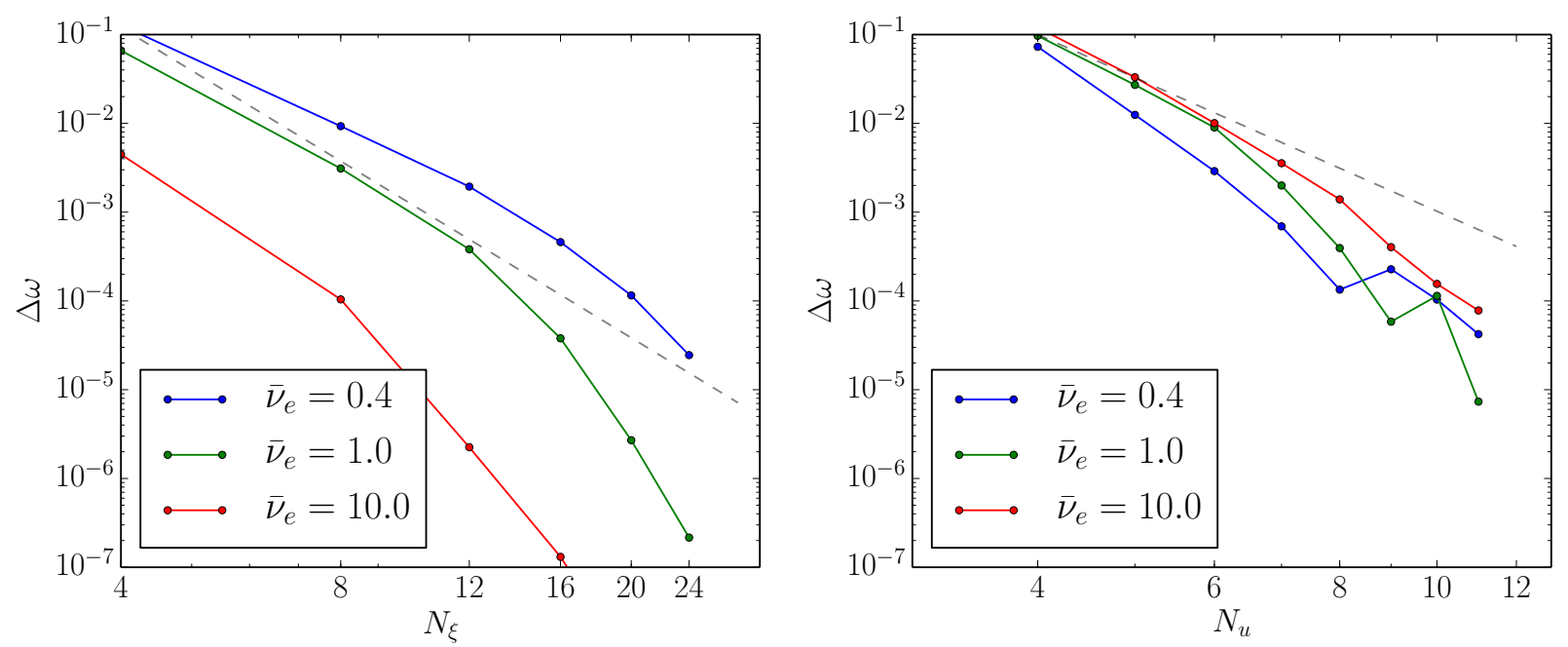

Figure 6: Convergence of the linear eigenmode frequency with respect to the number of pseudospectral meshpoints in the $\xi$ (left frame) and $u$ (right frame) dimensions, for different values of the collision frequency $\bar{\nu}_{e}$. In both cases, convergence is faster than $4^{\text {th }}$ order (dashed line).

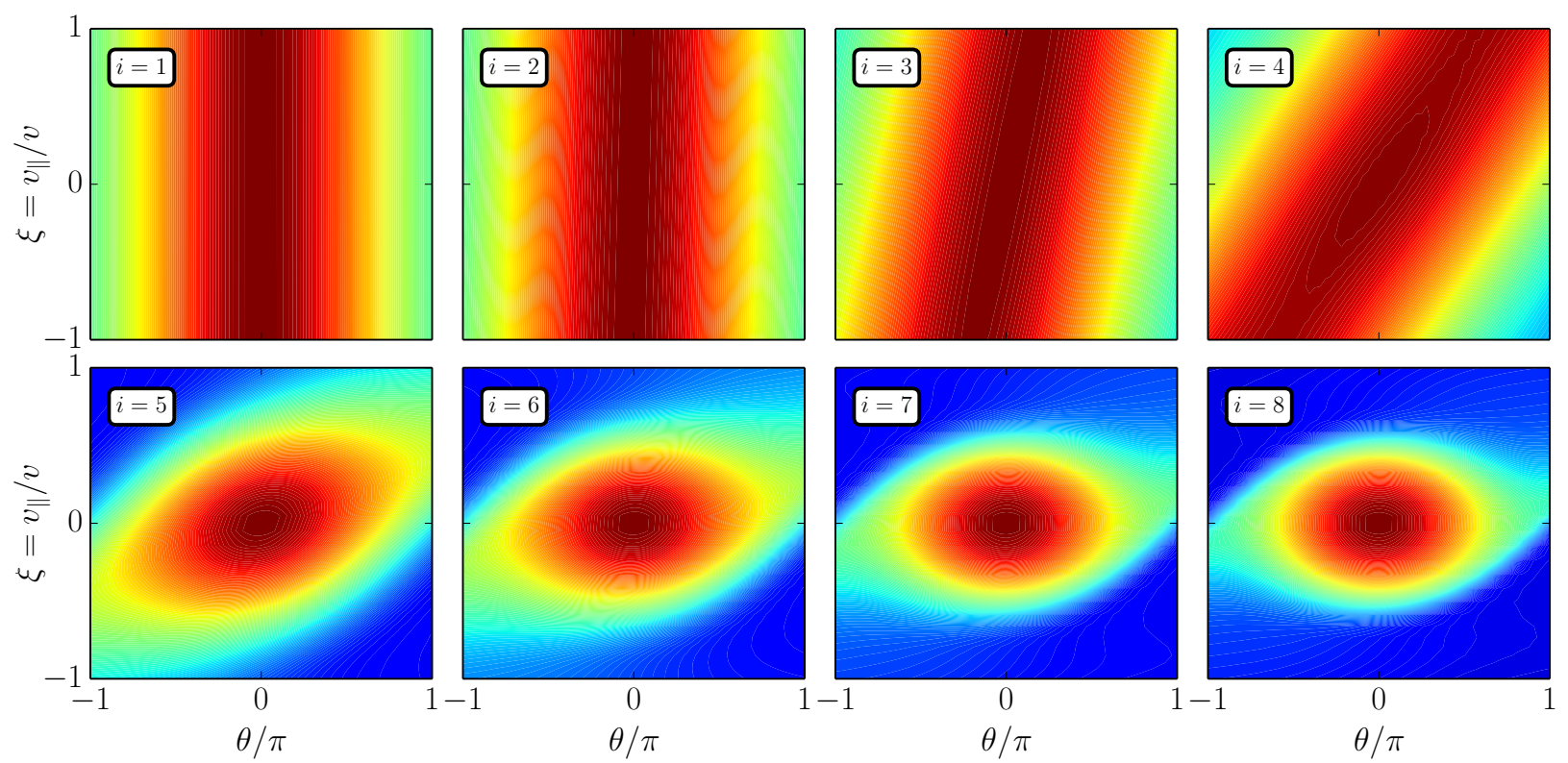

Figure 7: Absolute value of the complex electron distribution function, $\left|\tilde{H}_{e}\right|$, in the $(\theta, \xi)$-plane at different energy meshpoints. The transition from collisional to nearly collisionless regimes is clearly illustrated. Parameters for the $i^{\text {th }}$ mespoint are summarized in Table 1. 

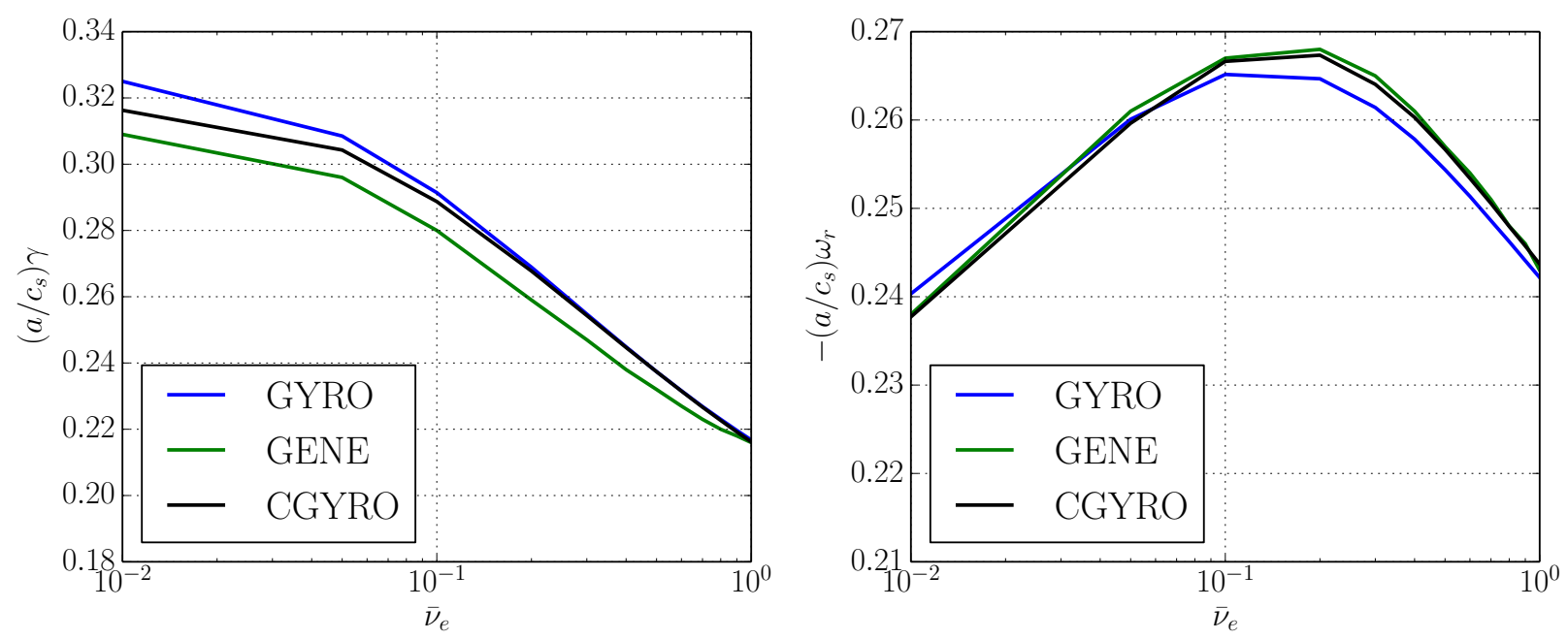

Figure 8: Linear growth rate $\gamma$ (left) and real frequency $\omega_{r}$ (right) versus dimensionless collision frequency, $\bar{\nu}_{e}$, for an ITG mode. Results from CGYRO are compared with GYRO and GENE.

unless $\bar{\nu}_{e}>1.0$. In the figure, Sugama DK refers to the drift kinetic limit $k_{\perp} \rightarrow 0$ of the Sugama operator. The results shows that these corrections are small but nonnegligible at large $\bar{\nu}_{e}$.

Next, Fig. 10 shows a comparison between CGYRO and the analytic theory of Rosenbluth and Hinton [38] for the residual zonal $(n=0)$ potential. Zonal flows are known to be an important mechanism to regulate ITG turbulence in nonlinear gyrokinetic simulations. In this standard test, an initial $n=0$ potential perturbation exhibits geodesic acoustic mode oscillations which decay collisionlessly by phase mixing. At long times the system decays to a steady-state residual potential such that the ratio $\Phi_{t=\infty} / \Phi_{t=0}$ can be calculated analytically. Using a very small value of $\epsilon=0.033$ ensures the analytic theory is very accurate, and close agreement with CGYRO is seen in Fig. 10a. It is well-known from analytic theory [39] that ion-ion collisions can reduce the residual zonal flow. The result is shown in Fig. 10b comparing CGYRO and GYRO. For this benchmark, rather than just the electron Lorentz operator, we also include the ion-ion Lorentz operator

$$
C_{i i}^{T} \simeq \nu_{i i}^{D}(v) \mathcal{L}
$$

in the ion gyrokinetic equation. When this is done, the codes show good agreement for the collisional damping rate.

\subsection{Nonlinear benchmarks}

We now move to more challenging benchmarks based on a nonlinear version of the GA standard case. All simulations retained 12 discrete complex toroidal modes $\left(N_{\alpha}=11\right)$,

$$
k_{\theta} \rho_{s}=0.0,0.07,0.14, \ldots, 0.77 .
$$

The radial and binormal box sizes were fixed for all cases at $L=114.3 \rho_{s}$ and $L_{y}=2 \pi / 0.07 \rho_{s} \simeq$ $89.8 \rho_{s}$, respectively. Other resolutions are $N_{r}=120, N_{\theta}=28, N_{u}=8, N_{\xi}=16$. The maximum energy was $b^{2}=8$. We remark that the maximum resolved wavenumber $k_{\theta} \rho_{s}=0.77$ is quite low and therefore truncates a large portion of the high-wavenumber tail of the electron spectrum. Yet, for this same reason, the case is a good choice for gauging how robustly the numerical algorithms deal with an under-resolved toroidal spectrum. All simulations are electromagnetic with a small but finite value of plasma pressure, $\beta_{e, \text { unit }}=0.05 \%$. Since CGYRO uses explicit time-integration 

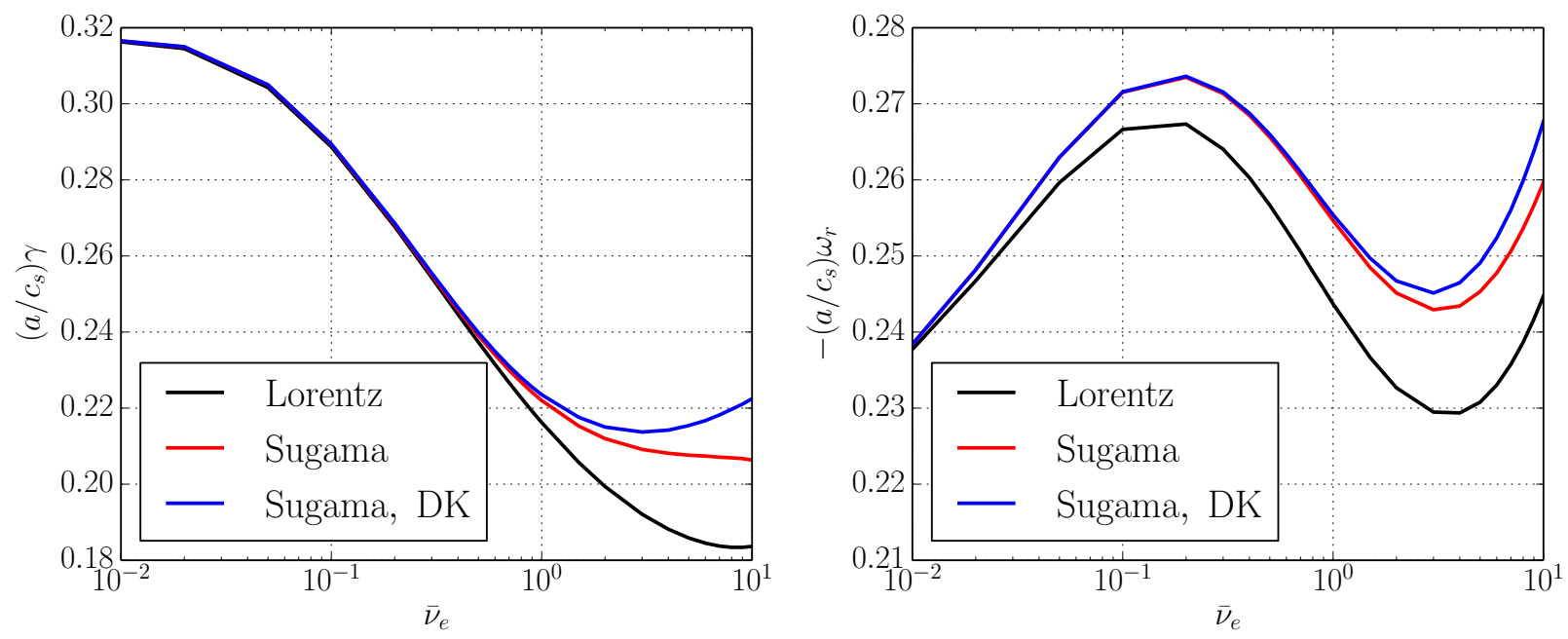

Figure 9: Linear simulations comparing relative linear growth rate $\gamma$ (and real frequency $\omega_{r}$ with Lorentz and Sugama collision operators in CGYRO. Sugama DK, or drift kinetic, refers to the Sugama operator in the limit $k_{\perp} \rightarrow 0$.
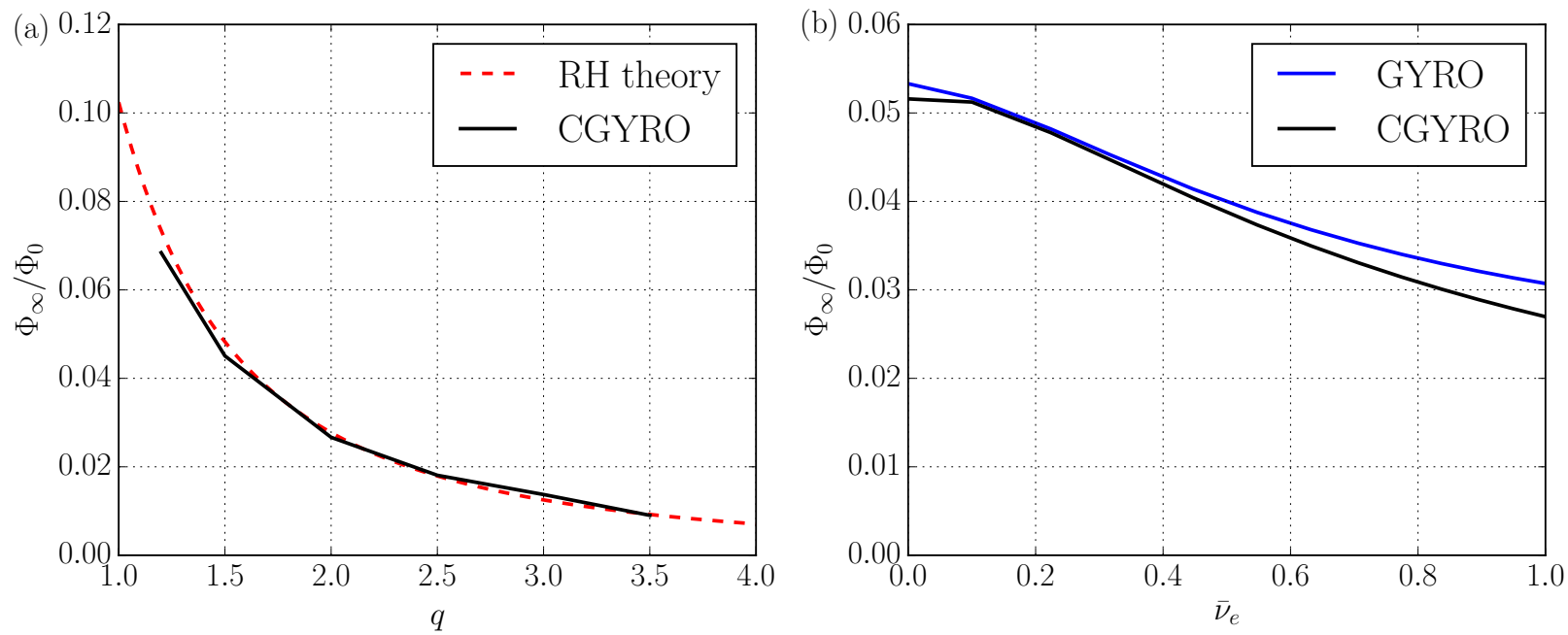

Figure 10: Residual zonal $(n=0)$ potential tests. Left frame (a) shows residual versus safety factor $q$ for a collisionless simulation with adiabatic electrons at $\epsilon=0.033$. The CGYRO residual agrees very closely with the Rosenbluth-Hinton analtyic theory. We have chosen a very small value of $\epsilon$ here to ensure that the theory is accurate. Right frame (b) shows residual versus collision rate $\bar{\nu}_{e}$ for the case of kinetic electrons with $\epsilon=0.167, q=2$. Note that this case includes both electron and ion Lorentz collisions. The CGYRO results agree closely with GYRO, with some divergence at very large collision frequency due to inaccuracy with the GYRO operator. 

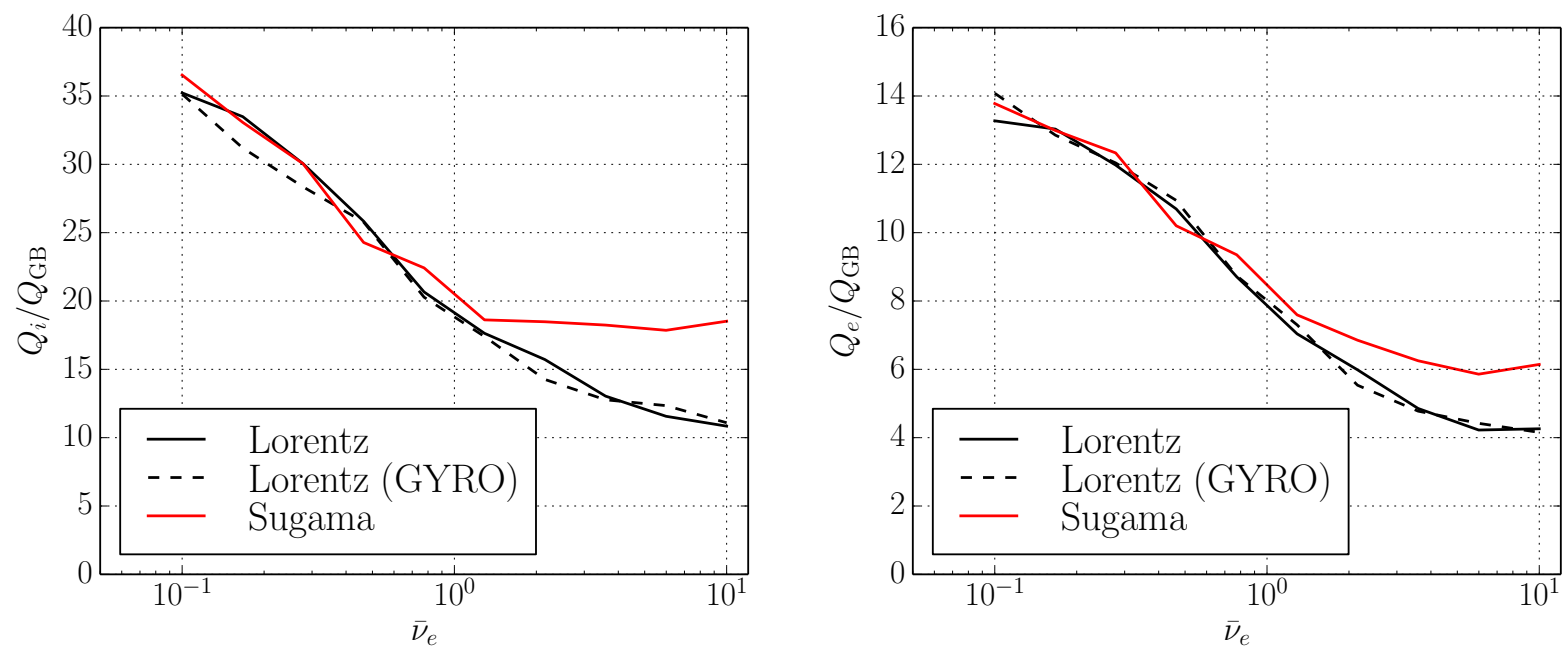

Figure 11: Nonlinear simulations comparing relative stabilization of energy transport with Lorentz and Sugama collision operators in CGYRO. For benchmarking purposes, GYRO results are shown as dashed curves.

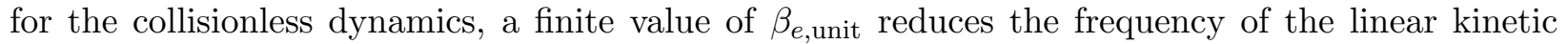
Alfvén wave and thus increases the maximum stable linear timestep.

As a preliminary investigation of the effect of the Sugama operator for a standard test case, we carry out a nonlinear $\bar{\nu}_{e}$-scan comparing the Lorentz and Sugama operators. This scan, which covers both the collisionless and strongly collisional regimes, is illustrated in Fig. 4.4. GYRO results are also shown to agree well with CGYRO-Lorentz results over the entire collisional range. We observe no statistically-significant difference between Lorentz and Sugama operators in the lowcollisionality region $\bar{\nu}_{e}<1$. However, in the high-collisionality region $\bar{\nu}_{e}>1$ we see an increasingly large discrepancy, with the Sugama operator predicting about 50\% larger energy fluxes by $\bar{\nu}_{e} \sim 10$. Note that the trend in energy flux closely matches the shape of the respective linear growth rates seen in Fig. 9. However, this collisionality is very high and seen only deep in the pedestal of modern large tokamaks. In a subsequent publication, we will show that for cases dominated by TEM instabilities, the discrepancy between operators can be larger than for this ITG-dominated case.

\section{Summary and Discussion}

A new electromagnetic gyrokinetic code, CGYRO, has been developed for accurate treatment of multispecies Fokker-Planck collisions including both pitch-angle and energy diffusion and finite Larmor radius $\left(k_{\perp}\right)$ effects. The primary motivations are $(1)$ more accurate collisions for outer plasma radii and modes like the TEM which are sensitive to collisions, and (2) the ability to do efficient nonlinear electromagnetic multiscale simulations up to $k_{\theta} \rho_{e}=1$. Discretization is based on $(\xi, v)$ velocity-space coordinates, with a Legendre-pseudospectral mesh in pitch angle, and a novel pseudospectral energy mesh derived from a set of nonstandard orthogonal polynomials. To our knowledge, this is the first pseudospectral implementation of the collision operator in a gyrokinetic code. In the two perpendicular spatial dimensions, the code is fully spectral with discrete wavenumber $\mathbf{k}_{\perp}$. The spectral method ensures the collision operator is algebraic (diagonal) in $k_{\perp}$, and for multiscale simulation in particular, spectral gyroaverages are the most efficient, requiring only a multiplication in wavenumber space. Given a spectral respresentation, the most 
efficient way to evaluate the nonlinear bracket is via FFT. Thus, the use of the dealiased FFT, coded entirely with FFTW, ensures maximum performance, portability and scalability of the relatively expensive nonlinearity. Finally, in the fieldline direction, a new 5th-order conservative upwind scheme is used to facilitate high-accuracy electromagnetic simulation even in the limit of very high plasma $\beta$ and vanishingly small perpendicular wavenumber, $k_{\perp} \rightarrow 0$. We show that the new solver agrees closely with GYRO in the limit of weak Lorentz collisions, but gives a significantly more realistic description of collisions at high collision frequency.

\section{Acknowledgements}

This material is based upon work supported by the U.S. Department of Energy, Office of Science, Office of Fusion Energy Sciences, Theory program and Center for the Study of Plasma Microturbulence under Award DE-FC02-08ER54963 and by the Edge Simulation Laboratory project under Grant DE-FC02-06ER54873.

[1] E. Belli, J. Candy, Full linearized Fokker-Planck collisions in neoclassical transport simulations, Plasma Phys. Control. Fusion 54 (2012) 015015.

[2] M. Barnes, I. Abel, W. Dorland, D. Ernst, G. Hammett, P. Ricci, B. Rogers, A. Schekochihin, T. Tatsuno, Linearized model Fokker-Planck collision operators for gyrokinetic simulations. II. Numerical implementation and tests, Phys. Plasmas 16 (2009) 072107.

[3] T. Görler, X. Lapillonne, S. Brunner, T. Dannert, F. Jenko, F. Merz, D. Told, The global version of the gyrokinetic turbulence code GENE, J. Comput. Phys. 230 (2011) 7053.

[4] P. Manas, Y. Camenen, S. Benkadda, W. Hornsby, A. Peeters, Enhanced stabilisation of trapped electron modes by collisional energy scattering in tokamaks, Phys. Plasmas 22 (2015) 062302 .

[5] E. Belli, J. Candy, Kinetic calculation of neoclassical transport including self-consistent electron and impurity dynamics, Plasma Phys. Control. Fusion 50 (2008) 095010.

[6] M. Rosenbluth, W. MacDonald, D. Judd, Fokker-Planck equation for an inverse-square force, Phys. Rev. 107 (1957) 1.

[7] J. Candy, R. Waltz, An Eulerian gyrokinetic-Maxwell solver, J. Comput. Phys. 186 (2003) 545.

[8] M. Kotschenreuther, G. Rewoldt, W. Tang, Comparison of initial value and eigenvalue codes for kinetic toroidal plasma instabilities, Comput. Phys. Commun. 88 (1995) 128.

[9] W. Dorland, F. Jenko, M. Kotschenreuther, B. Rogers, Electron temperature gradient turbulence, Phys. Rev. Lett. 85 (2000) 5579.

[10] J. Candy, E. Belli, GYRO Technical Guide, General Atomics Technical Report GA-A26818.

[11] F. Jenko, W. Dorland, M. Kotschenreuther, B. Rogers, Electron temperature gradient driven turbulence, Phys. Plasmas 7 (2000) 1904.

[12] A. Peeters, Y. Camenen, F. Casson, W. Hornsby, A. Snodin, D. Strintzi, G. Szepesi, The non-linear gyrokinetic flux tube code GKW, Comput. Phys. Commun. 180 (2009) 2650. 
[13] A. Brizard, Nonlinear gyrokinetic Maxwell-Vlasov equations using magnetic coordinates, J. Plasma Physics 41 (1989) 541.

[14] M. Kruskal, R. Kulsrud, Equilibrium of a magnetically confined plasma in a toroid, Phys. Fluids 1 (1958) 265.

[15] J. Candy, A unified method for operator evaluation in local Grad-Shafranov plasma equilibria, Plasma Phys. Control. Fusion 51 (2009) 105009.

[16] R. Waltz, R. Miller, Ion temperature gradient turbulence simulations and plasma flux surface shape, Phys. Plasmas 6 (1999) 4265.

[17] E. Belli, J. Candy, Fully electromagnetic gyrokinetic eigenmode analysis of high-beta shaped plasmas, Phys. Plasmas 17 (2010) 112314.

[18] E. Frieman, L. Chen, Nonlinear gyrokinetic equations for low-frequency electromagnetic waves in general plasma equilibria, Phys. Fluids 25 (1982) 502.

[19] H. Sugama, W. Horton, Nonlinear electromagnetic gyrokinetic equation for plasmas with large mean flows, Phys. Plasmas 5 (1998) 2560.

[20] E. Belli, J. Candy, An Eulerian method for the solution of the multi-species drift-kinetic equation, Plasma Phys. Control. Fusion 51 (2009) 075018.

[21] H. Sugama, T.-H. Watanabe, M. Nunami, Linearized model collision operators for multiple ion species plasmas and gyrokinetic entropy balance equations, Phys. Plasmas 16 (2009) 112503.

[22] I. G. Abel, M. Barnes, S. C. Cowley, W. Dorland, A. A. Schekochihin, Linearized model Fokker-Planck collision operators for gyrokinetic simulations. I. Theory, Phys. Plasmas 15 (2008) 122509.

[23] M. Rosenbluth, D. Ross, D. Kostomarov, Stability regions of dissipative trapped-ion instability, Nucl. Fusion 12 (1972) 3.

[24] D. Durran, Numerical methods for wave equations in geophysical fluid dynamics, SpringerVerlag, New York, 1999.

[25] T. Dannert, F. Jenko, Vlasov simulation of kinetic shear Alfvén waves, Comput. Phys. Commun. 163 (2004) 67.

[26] G. Golub, J. Welsch, Calculation of Gauss quadrature rules, Math. Comput. 23 (1969) 221.

[27] N. Steen, G. Byrne, E. Gelbard, Gaussian quadratures for the integrals $\int_{0}^{\infty} \exp \left(-x^{2}\right) f(x) d x$ and $\int_{0}^{b} \exp \left(-x^{2}\right) f(x) d x$, Math. Comp. 23 (1969) 661.

[28] M. Landreman, D. Ernst, New velocity-space discretization for continuum kinetic calculations and Fokker-Planck collisions, J. Comput. Phys. 243 (2013) 130.

[29] W. Gautschi, On the construction of Gauss quadrature rules from modified moments, Math. Comput. 24 (1970) 245.

[30] R. Sack, A. Donovan, An algorithm for Gaussian quadrature given modified moments, Numer. Math 18 (1972) 465. 
[31] F. Johansson, et al., mpmath: a Python library for arbitrary-precision floating-point arithmetic (version 0.18), http://mpmath.org/ (December 2013).

[32] S. Orszag, On the elimination of aliasing in finite-difference schemes by filtering highwavenumber components, J. Atmos. Sci. 28 (1971) 1074.

[33] M. Frigo, S. Johnson, The design and implementation of FFTW3, Proc. IEEE 93 (2) (2005) 216.

[34] R. Waltz, G. Staebler, W. Dorland, G. Hammett, M. Kotschenreuther, J. Konings, A gyrolandau fluid transport model, Phys. Plasmas 4 (1997) 2482.

[35] R. Miller, M. Chu, J. Greene, Y. Lin-liu, R. Waltz, Noncircular, finite aspect ratio, local equilibrium model, Phys. Plasmas 5 (1998) 973.

[36] J. Candy, C. Holland, R. Waltz, M. Fahey, E. Belli, Tokamak profile prediction using direct gyrokinetic and neoclassical simulation, Phys. Plasmas 16 (2009) 060704.

[37] J. Kinsey, R. Waltz, J. Candy, The effect of safety factor and magnetic shear on turbulent transport in nonlinear gyrokinetic simulations, Phys. Plasmas 13 (2006) 022305.

[38] M. Rosenbluth, F. Hinton, Poloidal flow driven by ion-temperature-gradient turbulence in tokamaks, Phys. Rev. Lett. 80 (1998) 724.

[39] F. Hinton, M. Rosenbluth, Dynamics of axisymmetric $(E \times B)$ and poloidal flows in tokamaks, Plasma Phys. Control. Fusion 41 (1999) A653. 